University of Rhode Island

DigitalCommons@URI

Open Access Master's Theses

2019

\title{
MOLECULAR MECHANISMS OF PROTEIN BINDING BY PERFLUOROALKYL SUBSTANCES (PFASS)
}

Michael Fedorenko

University of Rhode Island, mfedorenko@uri.edu

Follow this and additional works at: https://digitalcommons.uri.edu/theses

\section{Recommended Citation}

Fedorenko, Michael, "MOLECULAR MECHANISMS OF PROTEIN BINDING BY PERFLUOROALKYL SUBSTANCES (PFASs)" (2019). Open Access Master's Theses. Paper 1733.

https://digitalcommons.uri.edu/theses/1733

This Thesis is brought to you for free and open access by DigitalCommons@URI. It has been accepted for inclusion in Open Access Master's Theses by an authorized administrator of DigitalCommons@URI. For more information, please contact digitalcommons-group@uri.edu. 
MOLECULAR MECHANISMS OF PROTEIN BINDING BY

PERFLUOROALKYL SUBSTANCES (PFASs)

BY

MICHAEL FEDORENKO

A DISSERTATION SUBMITTED IN PARTIAL FULFILLMENT OF THE

REQUIREMENTS FOR THE DEGREE OF

MASTER OF SCIENCE

IN

CHEMICAL ENGINEERING

UNIVERSITY OF RHODE ISLAND

2019 
MASTER OF SCIENCE

$\mathrm{OF}$

MICHAEL FEDORENKO

\section{APPROVED:}

Thesis Committee:

Major Professor Geoffrey Bothun

Michael Greenfield

Rainer Lohmann

Nasser H. Zawia

DEAN OF THE GRADUATE SCHOOL

UNIVERSITY OF RHODE ISLAND

2019 


\begin{abstract}
Comparative investigation of the intermolecular chemical interaction (binding) between bovine serum albumin (BSA) and four polyfluoroalkyl substances (PFOA, PFNA, PFHxS, PFOS) by ${ }^{19}$ F NMR spectroscopy with synchronous observation of the ${ }^{19} \mathrm{~F}$ signals from both ends (head and tail) of the polyfluoroalkyl molecules was performed at three temperatures of $298 \mathrm{~K}, 304 \mathrm{~K}$ and $310 \mathrm{~K}$.

Chemical shifts of ${ }^{19} \mathrm{~F}$ NMR peaks in solutions of PFAS with BSA were used for evaluation of the dissociation constants, $\mathrm{K}_{\mathrm{d}}$, for both known mechanisms of PFAS binding with BSA: by hydrophobic interaction of the PFAS molecule carbon chain tail in the hydrophobic pockets of BSA ( $\Omega$ mechanism) and by hydrogen bond and electrostatic interaction of the PFAS molecule head group with charged regions on the BSA surface ( $\alpha$ mechanism). It was established that highest affinity of all four PFAS:BSA complexes is by the $\Omega$ mechanism of binding with $\mathrm{K}_{\mathrm{d}}$ at $310 \mathrm{~K}$ reaching values as low as $3.9 \times 10^{-6}, 6.5 \times 10^{-6}, 7.7 \times 10^{-6}$ and $1.9 \times 10^{-5} \mathrm{M}$ for PFOS, PFOA, PFNA, and PFHxS, in comparison with $5.7 \times 10^{-5}, 5.6 \times 10^{-5}, 6.6 \times 10^{-5}$ and $5.4 \times 10^{-5} \mathrm{M}$ values for $\alpha$ mechanism of binding.

Evaluation of the thermodynamic parameters (enthalpy $\Delta \mathrm{H}$, entropy $\Delta \mathrm{S}$, and Gibb's free energy $\Delta G$ ) showed that binding of PFOA, PFNA, PFHxS, and PFOS with BSA by both $\alpha$ and $\Omega$ mechanisms is accompanied by negative $\Delta H$ and $\Delta \mathrm{S}$ and positive $\Delta \mathrm{G}$ which are characteristic for binding of two large hydrophobic molecules with each other by weak hydrogen bond and van der Waals' forces.

$\mathrm{K}_{\mathrm{d}}$ for binding of the branched isopropyl isomers of PFHxS and PFOS with BSA were measured at $310 \mathrm{~K}$ as $8.8 \times 10^{-5}$ and $7.6 \times 10^{-5} \mathrm{M}$, correspondingly, which
\end{abstract}


indicated less affinity of isomers with the surface of BSA in comparison with the linear structure of PFHxS and PFOS molecules possibly due to "bulky" structure of the branched isomer head. 


\section{ACKNOWLEDGMENTS}

First, I would like to thank The Almighty God for giving me strength and wisdom to finish this degree, even at the most trying of times.

Secondly, my wife Abby for being my rock, helping me whenever I asked even with your own 3D printing project going on. Words cannot express how happy you make me and how proud I am of you. Thank you for your endless love and being the mother of my child. You were my patience and kindness.

This work would not have been finished without the help of my grandfather Dr. Anatoliy Fedorenko. He was a constant source of wisdom and provided the most valuable reference throughout the project. Your strive for excellence and to never stop learning has been an inspiration to me my entire life. I hope to one day finish the Ph.D. and to continue a life of hard work.

None of this would be possible without the dedication of my parents. You came to a country with no money, and selflessly built a home for Our family. You showed me what perseverance means and when to put the rest into faith and God. Thank you for everything. I hope one day I will get the chance to give back to you both.

To my Grandmother Sveta, sister Vera, brothers Dan and Paul and all of our family in Ukraine, thank you for giving me a strong self-identity. Through our unbreakable relationships I know who and what family truly is.

To Dr. Bothun, thank you for your vision and mentoring me. It has been a challenging and rewarding experience. I look forward to the future success of the URI STEEP group and that we continue to stay at the forefront of PFAS research. 


\section{PREFACE}

This basis for this research originally stemmed from my desire to help the environment. As consumer demands grow and new products are introduced to meet the needs of everyone, the ecological burden can go unnoticed compared to the convenience felt by the buyer. Researchers and scientists have a duty to determine the toxicity and environmental impacts of existing and new technologies and products. It is my passion to protect those who don't understand the complexity of the products sold to them and to prevent the abuse of the environment for economic gain.

I could not have achieved my current level of success without a strong support group. First of all, my family, who supported me with love and understanding. And

secondly, my committee members, each of whom has provided patient advice and guidance throughout the research process. Thank you all for your unwavering support.

This manuscript uses a Standard Format. 


\section{TABLE OF CONTENTS}

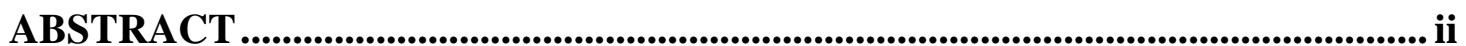

ACKNOWLEDGMENTS .......................................................................................... iv

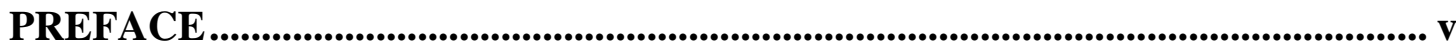

TABLE OF CONTENTS........................................................................................... vi

LIST OF TABLES ..................................................................................................................... vii

LIST OF FIGURES …....................................................................................................... viii

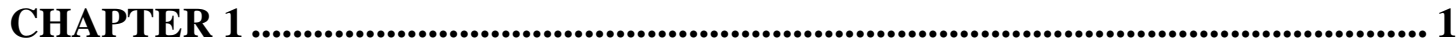

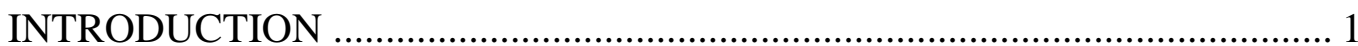

CHAPTER 2 …........................................................................................................................ 5

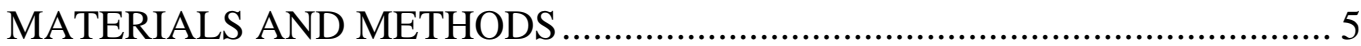

CHAPTER 3 ................................................................................................................................ 11

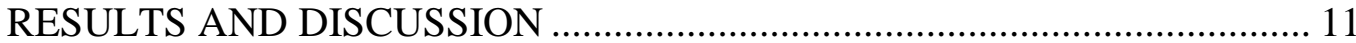

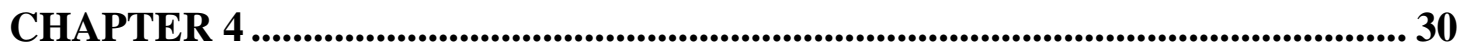

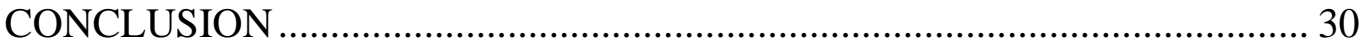

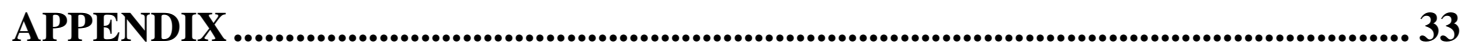

BIBLIOGRAPHY ................................................................................................................ 35 


\section{LIST OF TABLES}

TABLE

PAGE

Table 1. Thermodynamic parameters, enthalpy $(\Delta \mathrm{H})$, entropy $(\Delta \mathrm{S})$ and Gibb's free energy $(\Delta G)$ characterizing the interaction of PFOA, PFNA, PFHxS and PFOS with BSA in PBS buffer at pH 7.4 defined from linear van't Hoff plot only (Method 1) and

from the combination of van't Hoff plot and $K_{d}=e^{-\frac{\Delta G}{R T}}($ Method 2)................. 


\section{LIST OF FIGURES}

FIGURE

PAGE

Figure 1. Structures of PFOA (left) and PFOS (right) depicting the fluorines that are monitored in this CSP analysis and which binding mechanism ( $\alpha$ or $\Omega$ ) they

represent.

Figure 2. Exemplary spectra for (A) PFOA, (B) PFNA, (C) PFHxS and (D) PFOS at 1 $\mathrm{mM}$ in PBS solution in the absence of BSA....

Figure 3. Evolution of the $19 \mathrm{~F}$ peaks related to head $(\alpha)$ and tail $(\Omega)$ of PFOA with increasing PFOA concentration from $10 \mu \mathrm{M}$ to $1 \mathrm{mM}$ in absence (A) and presence (B) of BSA. 13

Figure 4. Dependence of the inverse chemical shift $(1 / \Delta \delta)$ of the $\alpha$ (circles) and $\Omega$ (squares) peaks on the concentration of PFOA (gray and black) and PFNA (yellow and red) measured at $298 \mathrm{~K}, 304 \mathrm{~K}$, and $310 \mathrm{~K} . \mathrm{R}^{2} \geq 0.95$ for PFOA and PFNA at all three temperatures. 15

Figure 5. Dependencies of the chemical shift, $\Delta \delta$, of $\alpha$ (circles) and $\Omega$ (squares) peaks on PFOA (orange) and PFNA (black) concentration with $10 \mu \mathrm{M}$ BSA measured at $310 \mathrm{~K}$ 16

Figure 6. Animated view of PFAS-BSA interaction following a two-step Langmuir sequence with the specific binding sites located in the protein hydrophobic core followed by the non-specific adsorption sites on the BSA surface. Texts in figure state the observed chemical shifts for the $\alpha$ and $\Omega$ peaks as the PFAS:BSA increases ....... 16 Figure 7. Evolution of the PFNA $\alpha$ and $\Omega$ peaks with increasing PFNA:BSA from 
7.5:1 to 100:1. Animated image displays the possible structure of the stabilized PFNA micelles due to the presence of BSA

Figure 8. Dissociation constants (Kd) for $\alpha$ (circles) and $\Omega$ (squares) mechanisms of binding of PFOA (orange) and PFNA (gray) with BSA measured at $298 \mathrm{~K}, 304 \mathrm{~K}$, and 310 K. Error bars are shown for each compound based on $n=3$.

Figure 9. Structure of the isopropyl isomers of PFHxS and PFOS determined by previous studies. ${ }^{23-25}$

Figure 10. Dependence of the inverse chemical shift (1/ $\Delta \delta$ ) of $\alpha$ (circles), $\Omega$ (squares) and I (triangles) peaks on concentration of PFHxS measured at $298 \mathrm{~K}, 304 \mathrm{~K}$, and 310 K. $\mathrm{R}^{2} \geq 0.95$ for PFHxS at all three temperatures. 21

Figure 11. Dependencies of the chemical shift, $\Delta \delta$, of $\alpha$ (circles), $\Omega$ (squares) and I (triangles) peaks on PFHxS concentration with $10 \mu \mathrm{M}$ BSA measured at 310K....... 21

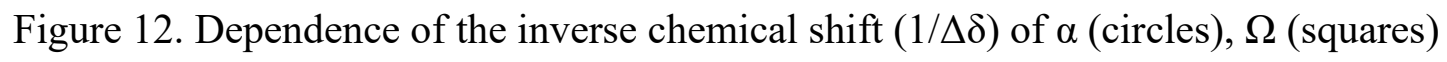
and I (triangles) peaks on concentration of PFOS measured at $298 \mathrm{~K}, 304 \mathrm{~K}$, and 310 K. $\mathrm{R}^{2} \geq 0.95$ for PFOS at all three temperatures 23

Figure 13. Dependencies of the chemical shift, $\Delta \delta$, of $\alpha$ (circles), $\Omega$ (squares) and I (triangles) peaks on PFOS concentration with $10 \mu \mathrm{M}$ BSA measured at $310 \mathrm{~K}$ 23

Figure 14. Dissociation constants (Kd) for $\alpha$ and $\Omega$ mechanisms of binding of PFHxS (green) and PFOS (blue) and their isopropyl isomers (I) binding with BSA measured at $298 \mathrm{~K}, 304 \mathrm{~K}$, and $310 \mathrm{~K}$. Error bars are shown for each compound based on $\mathrm{n}=3 \ldots 25$ Figure 15. Enthalpy $(\Delta \mathrm{H})$, entropy $(\Delta \mathrm{S})$ and free energy $(\Delta \mathrm{G})$ for the $\alpha$ and $\Omega$ mechanisms of binding for PFOA and PFNA with BSA from 298-310 K.. 26 Figure 16. Enthalpy $(\Delta \mathrm{H})$, entropy $(\Delta \mathrm{S})$ and free energy $(\Delta \mathrm{G})$ for the $\alpha, \Omega$ and I 
mechanisms of binding for PFHxS and PFOS with BSA from 298-310 K............... 27 


\section{CHAPTER 1}

\section{INTRODUCTION}

Since the end of the second World War, a group of synthetic fluorinated chemicals called per- and polyfluoroalkyl substances (PFASs) have been used broadly in numerous industrial and consumer applications to create non-adhesive surfaces and flame-resistant materials. ${ }^{1-4}$ PFASs are oleophilic with greater hydrophobicity and acidity than their hydrocarbon analogs. Due to the strength of the carbon-fluorine bond these compounds possess unique chemical and physical properties such as high chemical stability and thermal inertness, and ultra-low surface energy. These properties also lead to complex interactions within environmental and biological systems. ${ }^{1,2}$ PFASs persist in the environment, withstanding biodegradation, photolysis and hydrolysis. As a result, they bioaccumulate in the food chain, are transported long distances via air or water and are detected ubiquitously all over the world even in remote regions with no history of their production. ${ }^{1,2}$

Perfluorooctanoic acid (PFOA; $\mathrm{C}_{8} \mathrm{HF}_{15} \mathrm{O}_{2}$ ), perfluorononanoic acid (PFNA; $\left.\mathrm{C}_{9} \mathrm{HF}_{17} \mathrm{O}_{2}\right)$, perfluorohexanesulfonic acid $\left(\mathrm{PFHxS} ; \mathrm{C}_{7} \mathrm{HF}_{15} \mathrm{O}_{3} \mathrm{~S}\right)$ and perfluorooctanesulfonic acid (PFOS; $\mathrm{C}_{8} \mathrm{HF}_{17} \mathrm{O}_{3} \mathrm{~S}$ ) are four of the most historically used PFASs and have received particular interest among the thousands of different PFAS molecules due to their high frequency of detection in the environment and in humans. ${ }^{1,2,4}$ Studies have reported widespread exposure in humans, where PFASs were detected in the blood samples of over $99 \%$ of the individuals examined. ${ }^{5}$ They 
have also been found in cord serum of infants and in breast milk of nursing mothers. ${ }^{6,7}$ Their persistency is demonstrated by their long half-lives in humans, estimated to be 3.8 years for PFOA, 2.5 years for PFNA, 8.5 years for PFHxS, and 5.4 years for PFOS. ${ }^{1,8}$ From epidemiological studies, the critical effects of PFASs are an increase in serum total cholesterol in adults, ${ }^{9}$ a decrease in antibody response for vaccinations, ${ }^{10}$ pregnancy-induced hypertension and preeclampsia, ${ }^{11}$ and cancer. ${ }^{12,13}$ The mechanisms by which PFASs interact and transport throughout the human body are not well understood and are still being researched. ${ }^{10,14}$ These PFASs are detected primarily in the blood and the liver of humans, highlighting their proteinophilic nature. ${ }^{15}$

Human serum albumin (HSA), being a ligand binding protein, plays an important role in the accumulation pattern of PFASs in the blood and the liver tissue. ${ }^{16,17}$ The ability of a molecule to bind to HSA influences its lifetime and excretion from the body. ${ }^{18} \mathrm{HSA}$ at $0.6 \mathrm{mM}$ is the most abundant protein in humans, transporting different natural and exogenous ligands including fatty acids, pharmaceuticals and small organic anions throughout the human body. ${ }^{18}$ Studies have estimated that over $90 \%$ of the total PFASs in the body will be bound to HSA. ${ }^{19}$ With an aliphatic tail and anionic head group, PFASs are analogous to fatty acids and bind primarily with HSA due to its abundancy. ${ }^{20} \mathrm{HSA}$ contains seven distinct fatty acid binding sites that are asymmetrically distributed around the protein. ${ }^{18}$ Competition for binding sites between molecules can significantly affect the equilibrium between HSA-bound and HSA-unbound forms of the PFAS molecule. ${ }^{16,18}$

Dissociation constants, $\mathrm{K}_{\mathrm{d}}$, for PFAS-HSA binding reportedly range from $10^{-2}$ M to $10^{-6} \mathrm{M} \cdot{ }^{17,19,21}$ The range of $\mathrm{K}_{\mathrm{d}}$ over four orders of magnitude can be explained in 
part by the variety of experimental techniques amenable to different mechanisms of PFAS-HSA binding. ${ }^{22}$ HSA binds PFASs by two thermodynamic interactions: hydrophobic forces via the carbon tail in the hydrophobic pockets of HSA (specific binding) or by hydrogen bonds and electrostatic forces with the anionic head group in charged regions on the HSA surface (non-specific binding). Literature is conflicted on the mechanisms of PFAS-HSA binding and the number of binding sites on HSA. ${ }^{18-21}$ Fluorescence spectroscopy experiments have shown that PFASs interact with HSA specifically in hydrophobic cavities. These specific binding sites are sterically hindered and have a particular geometry that binds a limited number of PFAS molecules per protein. Studies using equilibrium dialysis report PFASs binding specifically and non-specifically to HSA. Once the specific sites with the higher binding affinity have been filled the PFASs will continue to be adsorbed nonspecifically throughout the charged regions on the surface of HSA with greater orientational freedom. Many PFASs are bound by non-specific adsorption because there is more available surface area than there are hydrophobic pockets. ${ }^{24}$ The adsorption phenomena can also help explain the number of PFAS binding sites on HSA ranging from 1 to 50 as described in literature..$^{20,25}$

Fatty acids have $K_{d}$ values in the same range as PFASs. ${ }^{18}$ Evaluating the potential for PFASs to displace fatty acids from HSA is important to understanding the detrimental impacts of PFASs on humans. A recent study has correlated PFASHSA $K_{d}$ values with placental transfer of PFASs in humans. Placental transfer efficiency (PTE) is the ratio of PFAS concentration in cord blood to that in maternal blood, which is an important marker in assessing fetal PFAS burden. ${ }^{23}$ 
The goal of this study was to investigate the binding mechanisms of four commonly used PFASs (PFOA, PFNA, PFHxS and PFOS) to bovine serum albumin (BSA) protein at relevant physiological parameters using fluorine nuclear magnetic resonance spectroscopy $\left({ }^{19} \mathrm{~F}\right.$ NMR $)$. BSA was used as a model protein as it closely resembles HSA and has similar binding sites. ${ }^{18}$ Fluorine NMR is a useful technique to characterize PFASs binding in biological mediums due to the unique ${ }^{19} \mathrm{~F}$ signal from the PFASs which are not generated by the protein. ${ }^{15,21,26-28}$ Comparative study of these four PFASs at the same experimental conditions with synchronous observation of the ${ }^{19} \mathrm{~F}$ signals from both the head and the tail of the PFAS molecules revealed how differences in charged head group and chain length impact PFAS binding strength and mechanisms to albumin protein. ${ }^{3,29}$ 


\section{CHAPTER 2}

\section{MATERIALS AND METHODS}

\section{$\underline{\text { 2.1. Chemicals }}$}

Bovine serum albumin (BSA) (99\% fatty acid free) lyophilized powder and trifluoromethyl acrylic acid (TFMAA) (98\%) solution were obtained from Sigma Aldrich (St. Louis, MO). Deuterium oxide $\left(\mathrm{D}_{2} \mathrm{O}\right)(99 \%)$ solution was purchased from Fisher Scientific (Agawam, MA). PFOA (99\%), PFNA (99\%), PFHxS (95\%) and PFOS (86\%) was obtained from Accustandard (New Haven, CT).

\subsection{Nuclear Magnetic Resonance Spectroscopy $\left({ }^{19} \mathrm{~F}\right.$ NMR)}

BSA concentration was held constant at $10 \mu \mathrm{M}$ in $\mathrm{pH} 7.4$ phosphate buffered saline (PBS) to apply chemical shift perturbation (CSP) analysis, which requires that PFAS be in in excess relative to the protein concentration. ${ }^{18,22}$ BSA solutions were prepared at least one day in advance of ${ }^{19} \mathrm{~F}$ NMR measurements and kept at $4{ }^{\circ} \mathrm{C}$ overnight. Each PFAS was dissolved in PBS and prepared at least a day in advance of the experiments. After dissolving in PBS, each sample vial was vortexed for 5 min to aid in dissolution and then stored at room temperature overnight. PFAS stock solutions were stored in polypropylene containers, which have been shown to adsorb less PFAS than glass containers. ${ }^{30}$ The PFAS stock solutions were diluted to achieve specific PFAS to BSA ratios. Then the PFAS solutions were transferred into analytical $5 \mathrm{~mm}$ NMR tubes at 90:10 PFAS: $\mathrm{D}_{2} \mathrm{O}$ ratios summing to $400 \mu \mathrm{l}$ of solution. $40 \mu \mathrm{l}$ of 
$\mathrm{D}_{2} \mathrm{O}$ solution were needed in every sample for proper NMR lock and calibration. $5 \mu \mathrm{l}$ of TFMAA was added as a second reference point for the CSP analysis. NMR spectra were obtained using a Bruker Advance III HD 400 NanoBay spectrometer (Bruker BioSpin, Rheinstetten, Germany) equipped with a $5 \mathrm{~mm}$ BBFO z-gradient smart probe using a Bruker Automatic Sample Changer (SampleXpress).

${ }^{19}$ F NMR spectra were automatically acquired under the control of ICONNMR (Bruker BioSpin, Rheinstetten, Germany) in the range from +20 to $-220 \mathrm{ppm}$ with the Bruker 5-mm auto-band probe tuned to $470 \mathrm{MHz}$ for ${ }^{19} \mathrm{~F}$ resonance. Chemical shifts were recorded relative to $\mathrm{D}_{2} \mathrm{O}(0.000 \mathrm{ppm})$ and TFMAA (-64.866 ppm). A $90^{\circ}$ pulse width for $18.0 \mu \mathrm{sec}$ was used for all experiments to provide the maximum signal to noise ratio $(\mathrm{S} / \mathrm{N})$ and to minimize the influence of the off-resonance effects on the accuracy of ${ }^{19}$ F NMR measurements. ${ }^{15} 4096$ scans were collected yielding 131072 data points to maximize the signal to noise ratio. $1 \mathrm{D}{ }^{19} \mathrm{~F}$ NMR spectra were obtained with a spectral width of $89285.7 \mathrm{~Hz}$, an acquisition time of $0.64 \mathrm{sec}$ and a recycle delay of $1 \mathrm{sec}$ to ensure full $\mathrm{T}_{1}$ relaxation. For evaluation of the thermodynamic parameters of the PFAS-BSA binding ${ }^{19} \mathrm{~F}$ NMR spectra were recorded at three different temperatures; 298K, 304K and 310K.

The Bruker pulse program, zgflqn, was used with a receiver gain (RG) of 212 . All ${ }^{19} \mathrm{~F}$ NMR spectra were automatically phased and baseline corrected for accurate quantitative measurements using the Topspin3.2 software package (Bruker BioSpin, Rheinstetten, Germany), MestReNova software package (Mestrelab Research, Escondido, CA) and Origin Software (Origin Lab, Northampton, MA). Peak shifts were obtained by the electronic Gaussian fit of the expanded regions around 
diagnostic resonances using Origin Software. All ${ }^{19} \mathrm{~F}$ NMR experiments were repeated independently in triplicate.

\subsection{Dissociation Constants $\left(\mathrm{K}_{\mathrm{d}}\right)$}

The dissociation constant, $\mathrm{K}_{\mathrm{d}}$, is an equilibrium constant that measures the propensity of a larger complex in the bound state to reversibly dissociate into its constituent parts. PFAS bound with BSA represents the large complex while unbound PFAS and BSA represent the smaller components. $K_{d}$ can be represented from the following reaction and ratio, seen in equation (1) and (2), and is inversely proportional to the association constant, $\mathrm{K}_{\mathrm{a}}$.

$$
\begin{gathered}
{[\mathrm{PFAS}-\mathrm{BSA}] \stackrel{\mathrm{K}_{\mathrm{d}}}{\Leftrightarrow}[\mathrm{PFAS}]+[\mathrm{BSA}]} \\
\mathrm{K}_{\mathrm{d}}=\frac{[\mathrm{PFAS}][\mathrm{BSA}]}{[\mathrm{PFAS}-\mathrm{BSA}]}
\end{gathered}
$$

The chemical shift of a ligand NMR signal in the presence of a protein is commonly used to monitor the formation of a protein-ligand complex. 1D NMR spectra of small-molecules ( $\mathrm{MW} \leq 500 \mathrm{Da}$ ) typically have sharp peaks due to a shorter dipole-dipole relaxation. Binding of a ligand to a high molecular weight molecule such as a protein induces peak broadening and a corresponding chemical shift in the NMR signal because the bound ligand experiences the slow relaxation time of the protein compared to the free state of the ligand. ${ }^{31}$

$K_{d}$ values were determined based on the resonance chemical shift, $\Delta \delta$, of the PFAS bound to the BSA relative to its unbound state in solution. ${ }^{19,20,26}$ Chemical shift perturbation (CSP) theory can determine the binding affinity of the ligand, the binding site(s) and the structure of the complex. ${ }^{28}$ The observed chemical shift is the 
population-weighted average of free and bound ligands, which allows the determination of $K_{d}$ from measurement of the peak positions. ${ }^{19,20,22,28}$ The chemical shift of the PFAS resonance peak is sensitive to structural differences of its bound and unbound states, meaning that a genuine binding interaction of PFAS with BSA will produce a perturbation. ${ }^{22,28} \mathrm{~A}$ CSP in excess of $0.02 \mathrm{ppm}$ indicates that the environmental structure of the ligand experiences some transformation (e.g. alteration of polarity, electrostatic interaction, etc.). ${ }^{22}$ At a fixed BSA concentration, these perturbations are dependent on the PFAS concentration, reflecting differences in the fraction of PFAS which is bound to BSA. A smaller fraction is bound at high PFAS concentrations, resulting in resonances that more closely resemble those of the free PFAS. These spectral changes are related to the fraction of bound ligand. ${ }^{32}$ $\mathrm{K}_{\mathrm{d}}$ values were determined graphically based on equation (3):

$$
[\mathrm{L}]_{\mathrm{T}}=\frac{\mathrm{n}[\mathrm{P}]_{\mathrm{T}}}{\Delta \delta} \cdot \Delta \delta_{\mathrm{B}_{\mathrm{app}}}-\mathrm{K}_{\mathrm{d}}
$$

where $\Delta \delta=\delta_{\mathrm{obs}}-\delta_{\text {free }}$ is the net chemical shift of the monitored resonance of the bound ligand, $[\mathrm{L}]_{\mathrm{T}}$ is the total ligand concentration, $[\mathrm{P}]_{\mathrm{T}}$ is the total concentration of protein, $\mathrm{n}$ is the number of binding sites per protein molecule and $\Delta \delta_{\text {Bapp }}$ is the apparent change in the chemical shift for the monitored resonance in the bound state. ${ }^{19,20}$ The value of $K_{d}$ is extracted as the negative $y$-intercept from the plot of the PFAS concentration versus the inverse of the PFAS chemical shift. ${ }^{19,20,22,33}$ By monitoring the perturbations of the chemical shifts for both the head and tail of the PFAS, it is proposed that the $\mathrm{K}_{\mathrm{d}}$ for both binding mechanisms (hydrophobic and electrostatic) can be evaluated. The monitored peaks arise from the fluoroethyl carbon adjacent to the anionic head group, labelled $\alpha$, representing the non-specific binding 
mechanism. And the fluoromethyl carbon at the end of the hydrophobic tail, labelled $\Omega$, representing the specific binding mechanism, shown in Figure 1 .
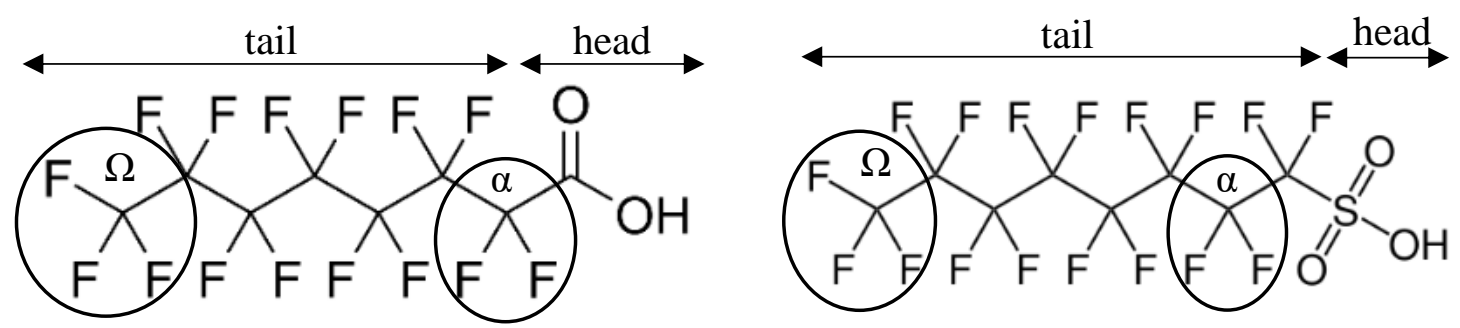

Figure 1: Structures of PFOA (left) and PFOS (right) depicting the fluorines that are monitored in this CSP analysis and which binding mechanism ( $\alpha$ or $\Omega$ ) they represent.

The $\mathrm{K}_{\mathrm{d}}$ values were measured for PFAS concentrations ranging from $10 \mu \mathrm{M}$ to $1 \mathrm{mM}$ for PFOA, PFNA, PFHxS and PFOS. This concentration range of PFAS is well above the $\sim 1 \mu \mathrm{M}$ detection limit of the ${ }^{19} \mathrm{~F}$ NMR method and can be found in a body of a highly exposed individual. ${ }^{1,27}$

\subsection{Thermodynamic Parameters of PFAS-BSA Binding}

Dissociation of PFAS with BSA is accompanied by a change of the Gibb's free energy, $\Delta \mathrm{G}$, that can be evaluated using equation (4):

$$
\mathrm{K}_{\mathrm{d}}=\mathrm{e}^{\frac{-\Delta \mathrm{G}}{\mathrm{RT}}}
$$

where $\mathrm{R}$ is the ideal gas constant and $\mathrm{T}$ is absolute temperature. For small temperature ranges the change in the enthalpy $\Delta \mathrm{H}$ and entropy $\Delta \mathrm{S}$ of a thermodynamic system are essentially constant and equation (5) can be used for $\Delta \mathrm{G}$ without the need to take into account the temperature dependencies of $\Delta \mathrm{H}$ and $\Delta \mathrm{S}$ :

$$
\Delta \mathrm{G}=\Delta \mathrm{H}-\mathrm{T} \Delta \mathrm{S}
$$


$\Delta \mathrm{H}$ and $\Delta \mathrm{S}$ are determined from the slope $(-\Delta \mathrm{H} / \mathrm{R})$ and the $\mathrm{y}$-intercept $(\Delta \mathrm{S} / \mathrm{R})$ based on the van't Hoff equation (6):

$$
\ln \left(\mathrm{K}_{\mathrm{d}}\right)=\frac{-\Delta \mathrm{H}}{\mathrm{RT}}+\frac{\Delta \mathrm{S}}{\mathrm{R}}
$$

The signs of $\Delta \mathrm{H}$ and $\Delta \mathrm{S}$ (+ or -) determine the dominant intermolecular forces for PFAS-BSA binding: hydrophobic interactions when $\Delta \mathrm{H}>0$ and $\Delta \mathrm{S}>0$, electrostatic interactions when $\Delta \mathrm{H}<0$ and $\Delta \mathrm{S}>0$, or van der Waals interactions and hydrogen bonding when $\Delta \mathrm{H}<0$ and $\Delta \mathrm{S}<0 .{ }^{34-37}$

The effect of carbon chain length and head group structure on the thermodynamic mechanisms of PFAS-BSA binding is analyzed in this comparative study of two carboxylates (PFOA and PFNA) and two sulfonates (PFHxS and PFOS) in solutions with BSA. 


\section{CHAPTER 3}

\section{RESULTS AND DISCUSSION}

\subsection{PFAS Characterization}

Figure 2 shows the chemical structures and associated $1 \mathrm{D}{ }^{19} \mathrm{~F}$ NMR spectra for PFOA, PFNA, PFHxS and PFOS in PBS in the absence of BSA. PFOA and PFNA exhibited clean spectra with no impurities or branched isomers detected, similar to

prior ${ }^{19} \mathrm{~F}$ NMR results. ${ }^{15,19,20}$ In contrast, PFHxS and PFOS contained impurities and branched isomers, causing the signal intensity to decrease and the noise to increase. PFASs are industrially manufactured on a large scale using the Simons Electrochemical Fluorination (ECF) process. With the longer chain sulfonates, such as PFOS, fragmentation and rearrangement of the carbon skeleton occurs during this process, producing a mixture of linear and branched isomers as well as shorter chain homologues. The ECF of short-chain sulfonates gives significantly better percentage of the linear compounds. ${ }^{33,38,39}$ This is confirmed in Figure 2; the $1 \mathrm{D}{ }^{19} \mathrm{~F}$ NMR spectra for PFHxS and PFOS exhibited roughly 5\% and 30\% branched isomers based on peak integration of the linear and branched peaks. Understanding how isomers differ in binding to HSA has not been studied in detail due to challenges connected with the coalescing and splitting of isomer peaks. ${ }^{38}$ 

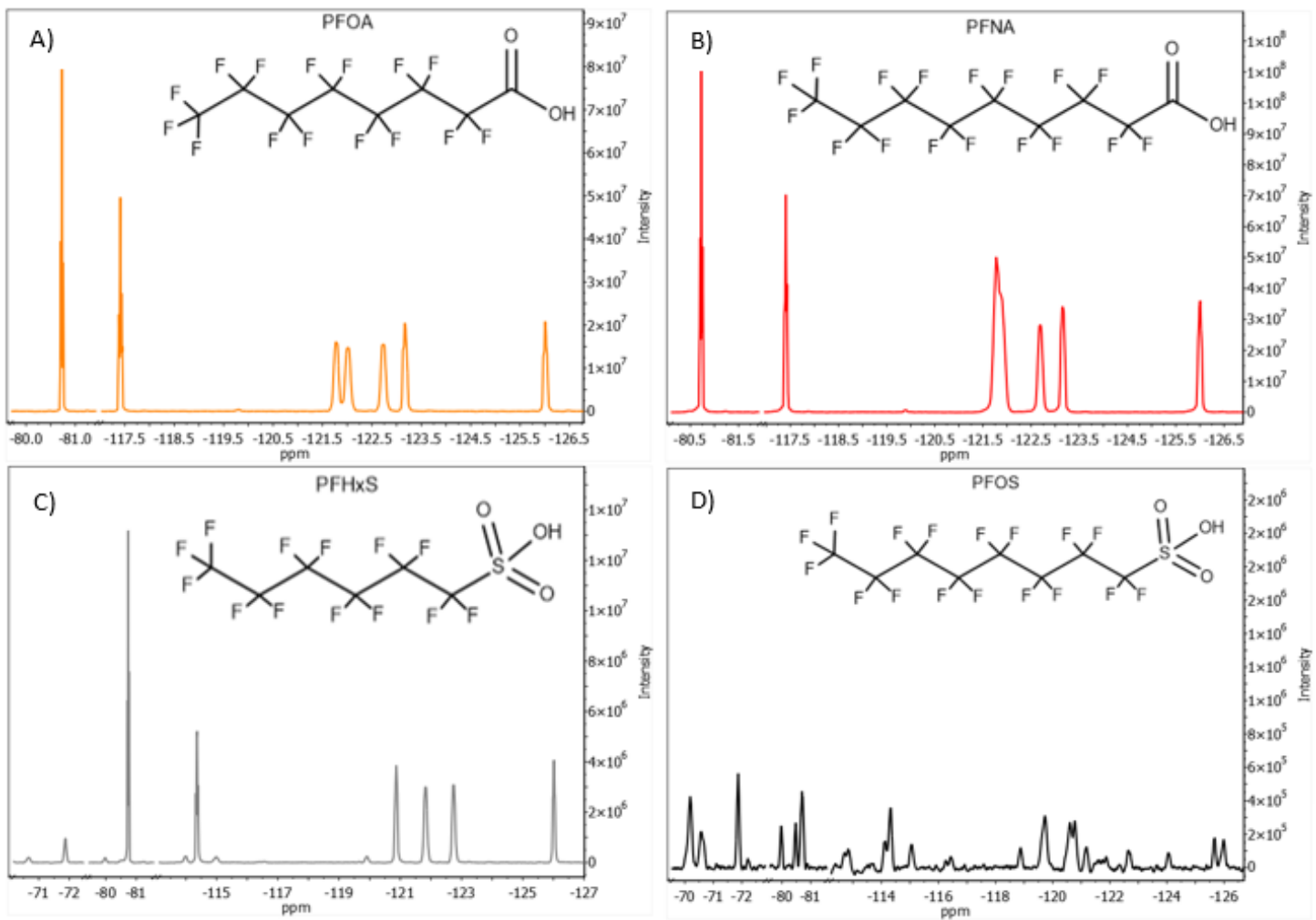

Figure 2: Exemplary spectra for (A) PFOA, (B) PFNA, (C) PFHxS and (D) PFOS at 1 $\mathrm{mM}$ in PBS solution in the absence of BSA.

\subsection{Dissociation Constants $\left(\mathrm{K}_{\mathrm{d}}\right)$ and Binding Mechanisms}

${ }^{19} \mathrm{~F}$ NMR spectroscopy observation is a powerful tool to study protein-ligand interactions because each fluorine atom gives an individual signal in the spectrum that carries information on the local chemical environment. ${ }^{(22,40)}$ An advantage of ${ }^{19} \mathrm{~F}$ NMR spectroscopy is that it can measure $\mathrm{K}_{\mathrm{d}}$ in the $\mu \mathrm{M}$ range which is not well covered by traditional biochemical binding assays. ${ }^{22}$ The following criteria are required to identify PFAS binding mechanisms: (1) the molecular recognition event is sufficiently defined to provide a well-structured binding complex; (2) there are a number of independently varying ${ }^{19} \mathrm{~F}$ NMR signals that shift, providing a multidimensional analysis; and (3) the shift of the PFAS ${ }^{19} \mathrm{~F}$ resonances is induced by spatial proximity to the protein to access structural information on the whole PFAS 
molecule. ${ }^{41}$ By comparing the chemical shifts for both ends of a PFAS molecule, the 1D ${ }^{19} \mathrm{~F}$ NMR experiments can provide insight into the binding mechanism and structure of the PFAS bound to BSA. ${ }^{19,20,21,25}$

\subsubsection{Carboxylates (PFOA and PFNA)}

PFOA and PFNA exhibited significant ${ }^{19} \mathrm{~F}$ chemical shift and broadening in both $\alpha$ and $\Omega$ resonances upon binding to BSA. Figure 3 shows the evolution of the PFOA peaks corresponding to the ${ }^{19} \mathrm{~F}$ atoms located on the carbon near the head $(\alpha)$ and the final carbon on the tail $(\Omega)$ of the compound, with increases in concentration from $10 \mu \mathrm{M}$ up to $1 \mathrm{mM}$ in the absence (Figure $3 \mathrm{~A}$ ) and in the presence (Figure 3B) of BSA. Without protein, $\alpha$ and $\Omega{ }^{19} \mathrm{~F}$ resonances did not shift nor broaden with increasing PFAS concentration (Figure 3A). With BSA present, these peaks shifted and broadened reflecting PFAS-BSA complex formation (Figure 3B). This is most evident at low PFOA concentrations (i.e. low [PFOA:BSA] ratios) where almost all measurable PFOA is protein-bound, and the $\alpha$ and $\Omega{ }^{19} \mathrm{~F}$ resonances are perturbated the farthest from the original BSA free positions. With an increase in the PFAS concentration in solution with BSA, $\alpha$ and $\Omega{ }^{19} \mathrm{~F}$ resonances return their shifts on the spectrum in solution without BSA as shown in Figure 3. The fluorine $\alpha$ resonance on the carbon adjacent to the headgroup is the most sensitive to protein binding and does not fully return to its original position, similar to results from prior studies. ${ }^{20}$ 

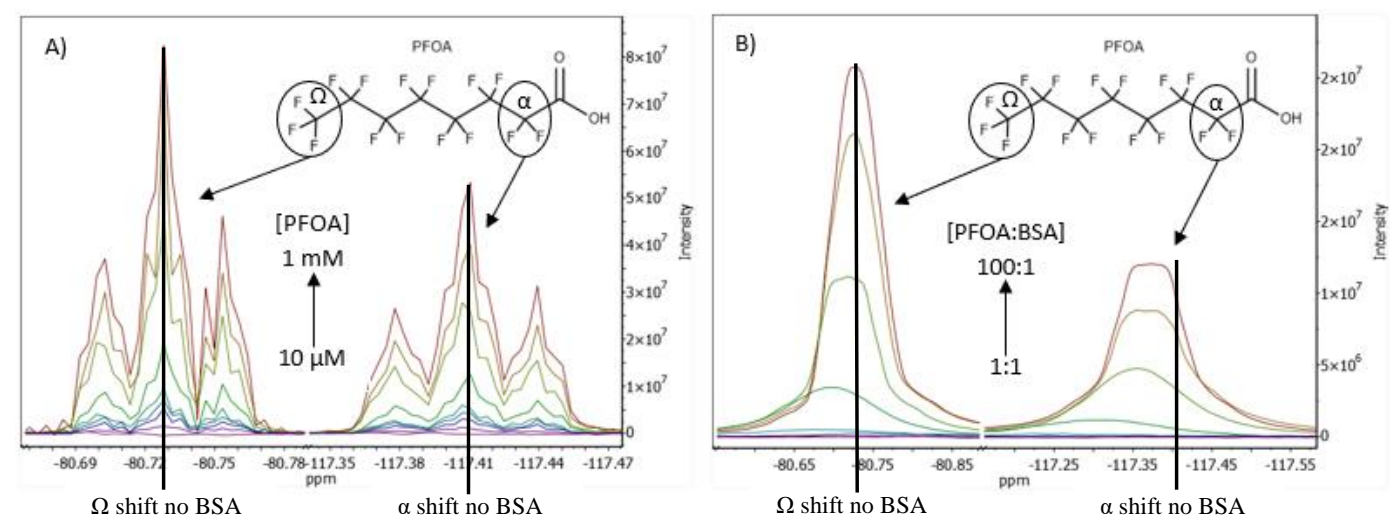

Figure 3: Evolution of the ${ }^{19} \mathrm{~F}$ peaks related to head $(\alpha)$ and tail $(\Omega)$ of PFOA with increasing PFOA concentration from $10 \mu \mathrm{M}$ to $1 \mathrm{mM}$ in absence (A) and presence (B) of BSA.

The change in the PFAS chemical shift is an average of specific $(\Omega)$ and nonspecific binding $(\alpha)$ with the protein. ${ }^{31}$ By analyzing the ${ }^{19} \mathrm{~F}$ signals from opposite ends (head $(\alpha)$ and tail $(\Omega)$ ) of the PFAS it is possible to distinguish between specific and nonspecific binding. Figure 4 and 5 show that both carboxylates, PFOA and PFNA, follow this pattern of the $\alpha$ and $\Omega$ peaks shifting on the NMR spectrum in solutions with $10 \mu \mathrm{M}$ BSA at three different temperatures, 298K, 304K, and 310K. As the PFAS concentration increases both the $\alpha$ and $\Omega$ peaks return to their original positions on the spectrum without BSA, indicating a saturation of the specific and non-specific binding sites.

The $\alpha$ and $\Omega$ peaks were not detected below 2.5:1 for PFOA:BSA or below 7.5:1 for PFNA:BSA, suggesting that below these ratios all PFASs are bound to protein. As seen in Figure 5, there is a significant chemical shift of the $\alpha$ peak across the entire PFOA and PFNA concentration range that demonstrates that the majority of the PFAS molecules adsorb non-specifically via electrostatic interactions. At the same time, the return of the $\Omega$ peak chemical shift with an increase of PFOA or PFNA 
concentration suggests that only a small quantity of the PFAS molecules, much below a ratio of 25:1 PFAS:BSA, bind specifically with BSA through the hydrophobic interaction of the PFAS carbon tail, as seen in Figure 5. These observations are consistent with previous studies of PFAS-BSA binding which established the existence of up to 50 sites on the BSA molecule surface available for $\alpha$-type binding and a few hydrophobic pockets available for $\Omega$-type binding in the BSA molecule core. ${ }^{17,20,25}$ Studies employing fluorospectrometry, isothermal titration calorimetry and circular dichroism have shown that the PFAS-BSA interaction follows a two-step Langmuir sequence and that the favorite binding site is located in the protein hydrophobic core. ${ }^{42}$ This two-step binding mechanism is supported by the data found in this study shown in Figure 5 and in Figure S1 (see supplementary material in Appendix A). An animated illustration of this two-step sequence is shown in Figure 6.

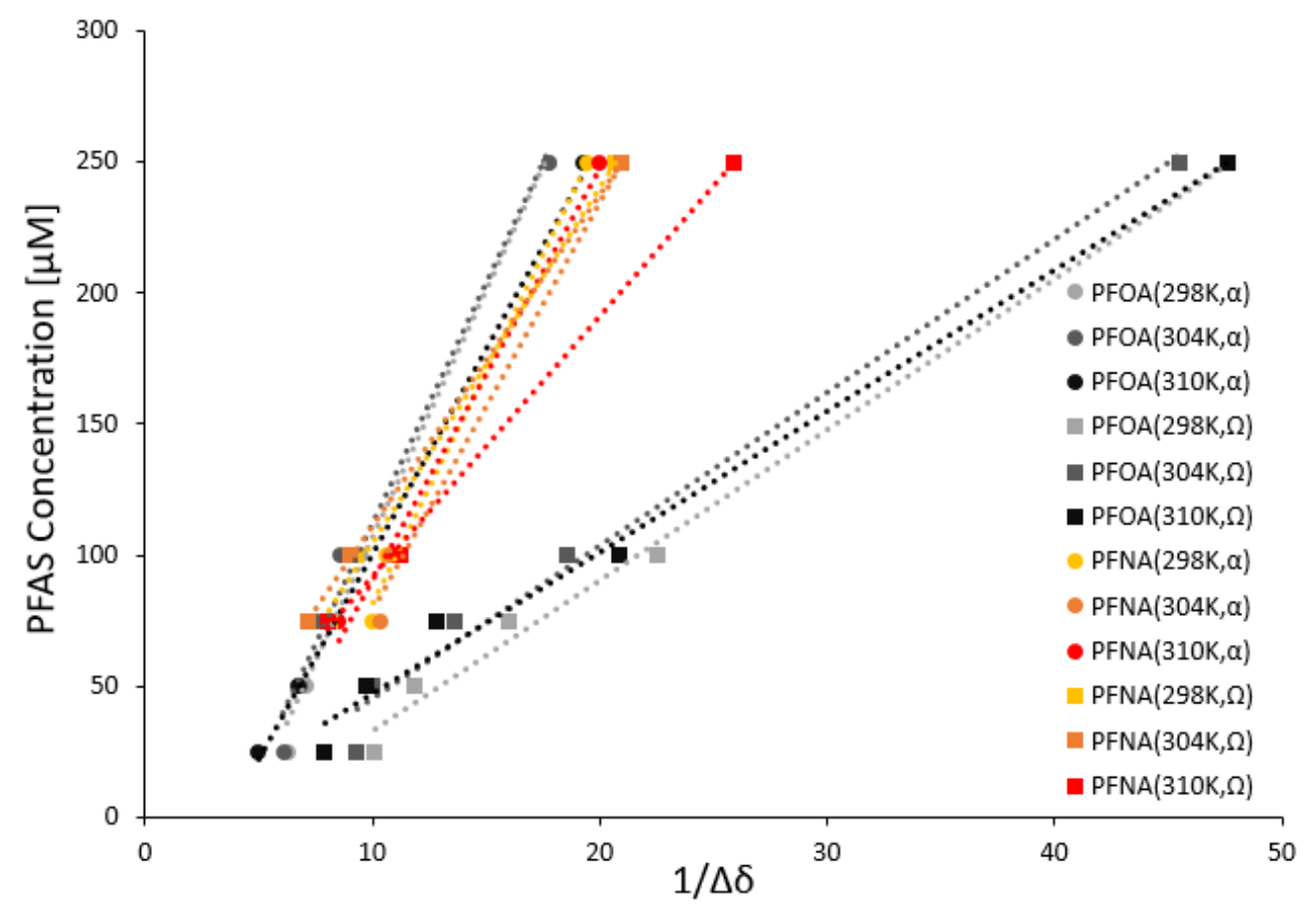

Figure 4: Dependence of the inverse chemical shift $(1 / \Delta \delta)$ of the $\alpha$ (circles) and $\Omega$ (squares) peaks on the concentration of PFOA (gray and black) and PFNA (yellow and red) measured at $298 \mathrm{~K}, 304 \mathrm{~K}$, and $310 \mathrm{~K} . \mathrm{R}^{2} \geq 0.95$ for PFOA and PFNA at all three temperatures. 


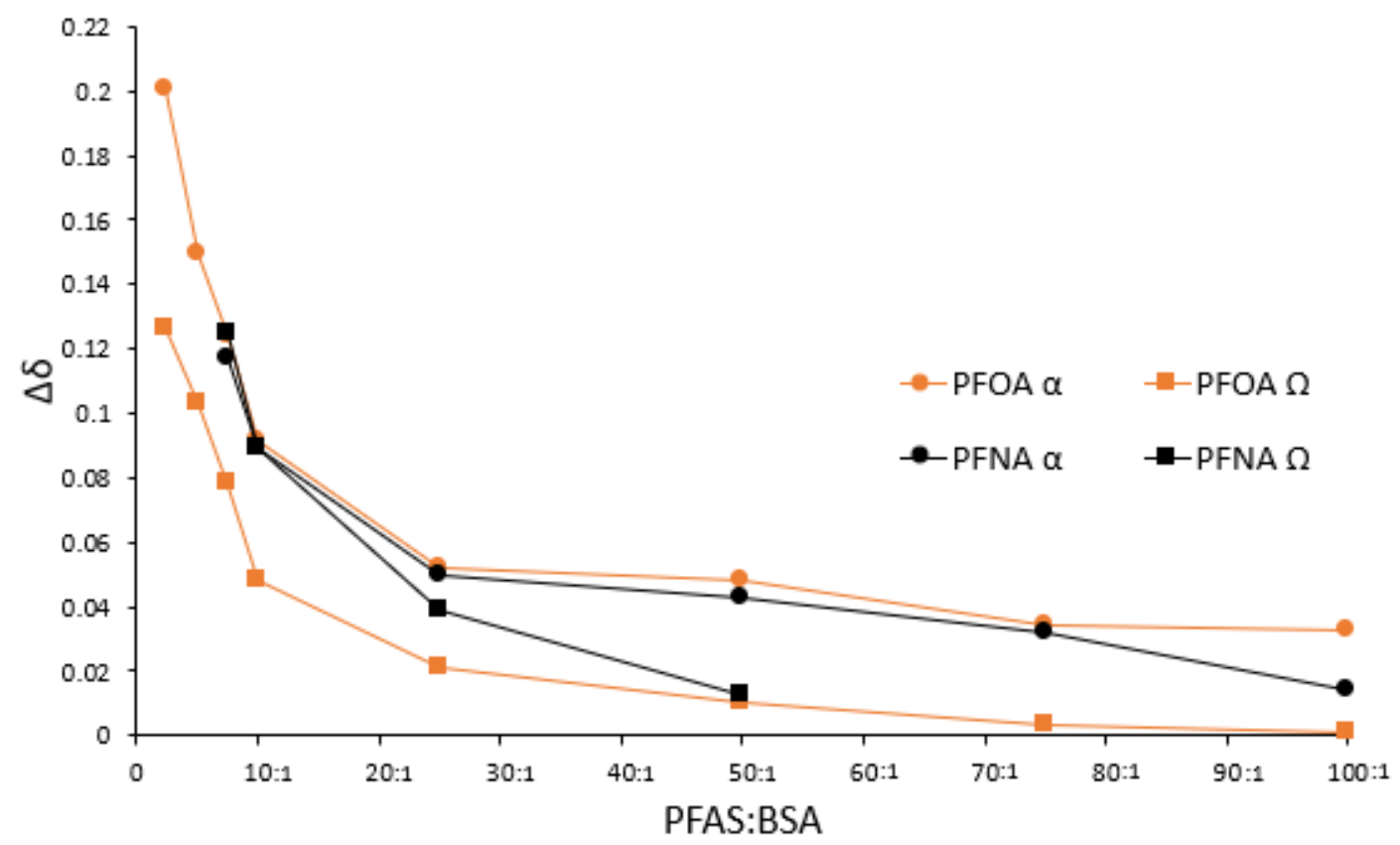

Figure 5: Dependencies of the chemical shift, $\Delta \delta$, of $\alpha$ (circles) and $\Omega$ (squares) peaks on PFOA (orange) and PFNA (black) concentration with $10 \mu \mathrm{M}$ BSA measured at $310 \mathrm{~K}$.

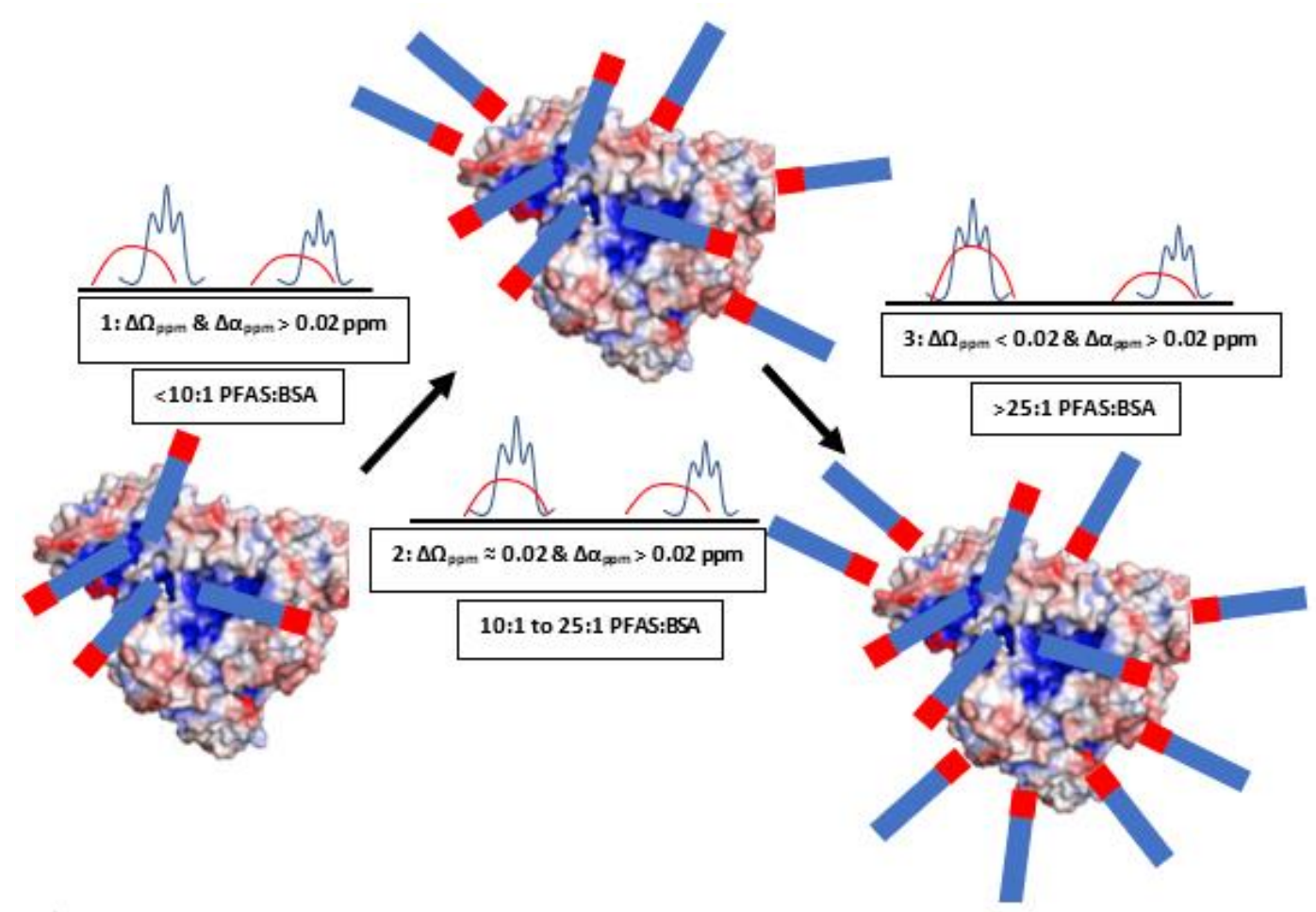

Figure 6: Animated view of PFAS-BSA interaction following a two-step Langmuir sequence with the specific binding sites located in the protein hydrophobic core followed by the non-specific adsorption sites on the BSA surface. Texts in figure state the observed chemical shifts for the $\alpha$ and $\Omega$ peaks as the PFAS:BSA increases. 
The PFNA $\Omega$ peak returned to its original position at a 50:1 PFNA:BSA and then continued to shift upfield at higher ratios (Figure 7). This was the only compound to have this occur at high PFNA:BSA ratios, and may reflect BSA promoting the formation of adsorbed PFNA micelles, possibly through hydrogen bond formation and electrostatic interactions of the anionic head group and the charged surface area of BSA. This stabilization effect could explain the upfield shift and disappearance of the PFNA $\Omega$ peak. This suggests that the addition of BSA disrupts normal surfactant behavior, possibly through the formation of surfactant-protein aggregates, shown by previous studies. ${ }^{19}$

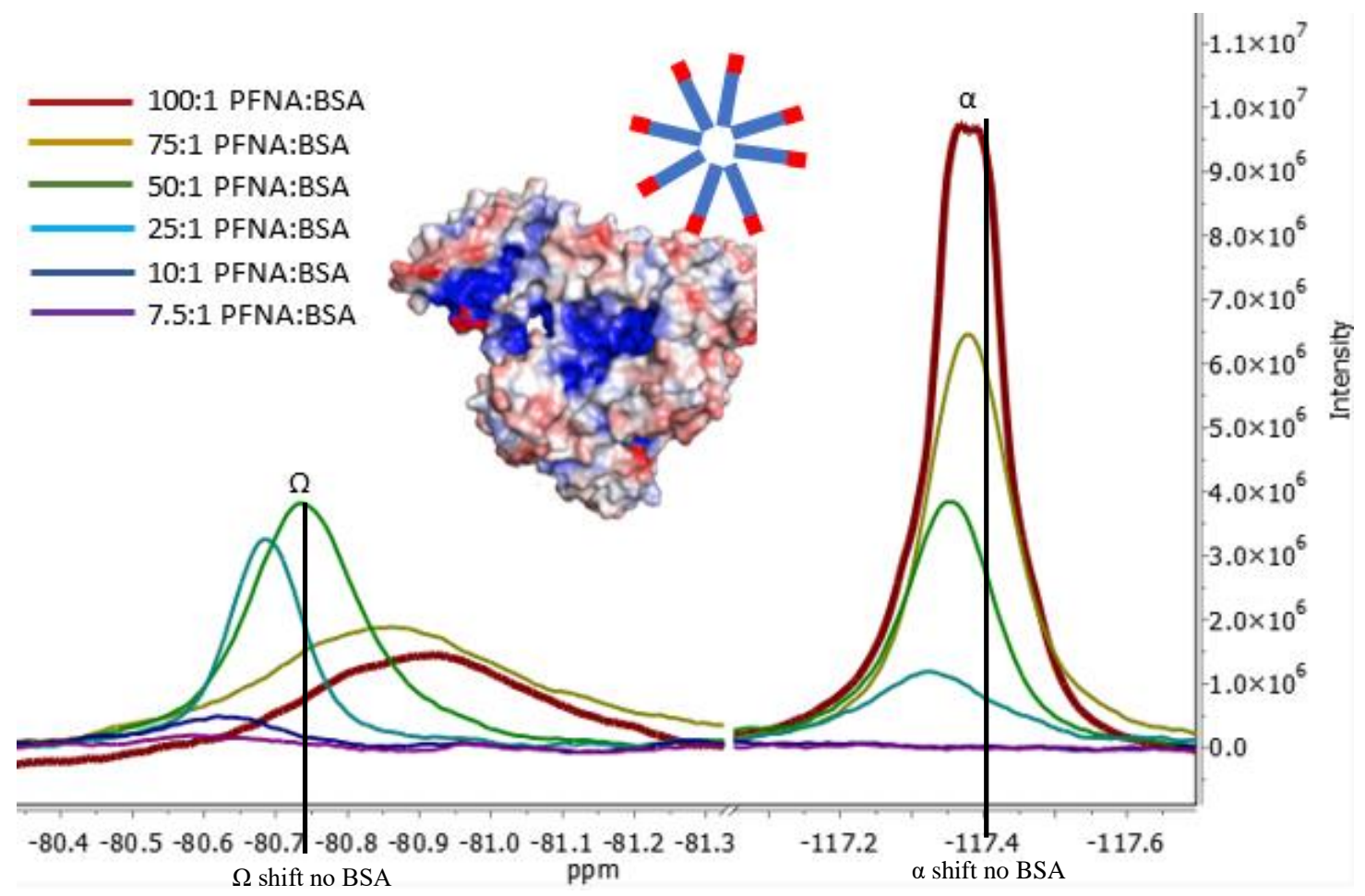

Figure 7: Evolution of the PFNA $\alpha$ and $\Omega$ peaks with increasing PFNA:BSA from 7.5:1 to 100:1. Animated image displays the possible structure of the stabilized PFNA micelles due to the presence of BSA.

Figure 8 shows the graphically determined $\mathrm{K}_{\mathrm{d}}$ for the $\alpha$ and $\Omega$ mechanisms of PFOA and PFNA binding with BSA using measured chemical shifts, $\Delta \delta$, and equation 
(1). Both $\alpha$ and $\Omega$ binding mechanisms have a $K_{d}$ in the $10^{-5} \mathrm{M}$ range. At $310 \mathrm{~K}$, the hydrophobic interaction of the carbon tail for PFOA and PFNA with the BSA core have a $\mathrm{K}_{\mathrm{d}}$ near $10^{-6} \mathrm{M}$. Having a $\mathrm{K}_{\mathrm{d}}$ this low makes PFOA and PFNA ligand competitors with natural fatty acids to form complexes with BSA. For both PFOA and PFNA, $K_{d}$ decreased with increasing temperature. Indicating that hydrophobic forces are the strongest in the formation of PFAS:BSA complexes. PFOA is characterized by a lower $\mathrm{K}_{\mathrm{d}}$ at all three temperatures compared to PFNA, suggesting that PFOA has a stronger affinity for BSA than PFNA due to steric interactions. PFASs with longer carbon tails can adopt helical twists that sterically hinder binding to the hydrophobic pockets of BSA. ${ }^{43}$

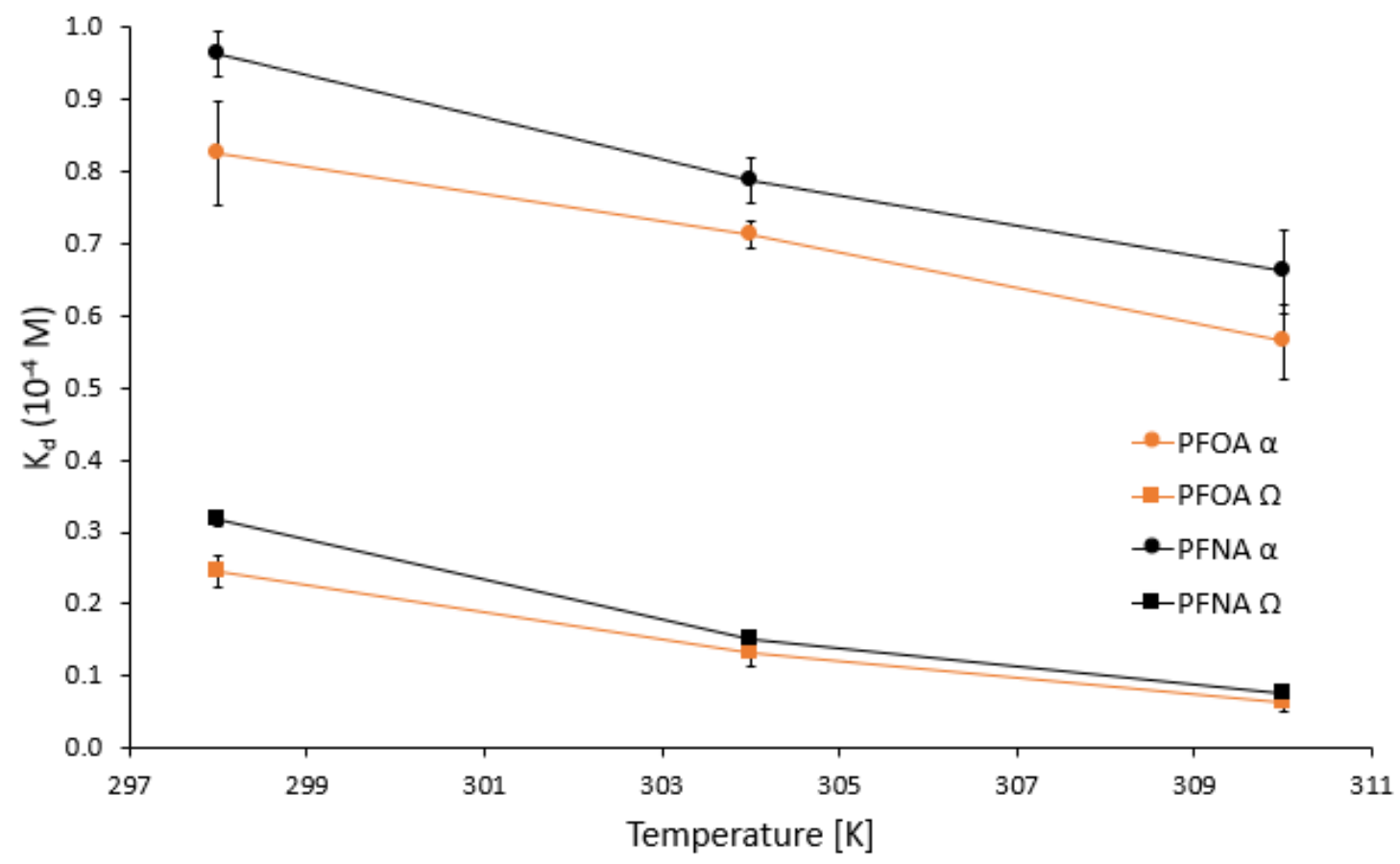

Figure 8: Dissociation constants $\left(\mathrm{K}_{\mathrm{d}}\right)$ for $\alpha$ (circles) and $\Omega$ (squares) mechanisms of binding of PFOA (orange) and PFNA (gray) with BSA measured at $298 \mathrm{~K}, 304 \mathrm{~K}$, and 310 K. Error bars are shown for each compound based on $n=3$. 


\subsubsection{Sulfonates (PFHxS and PFOS)}

PFOS ${ }^{19}$ F NMR peaks had almost two orders of magnitude lower signal intensity in comparison with the other three PFASs with or without BSA due to the significant content of isomers $(\sim 30 \%) .{ }^{28}$ PFHxS exhibited less isomers content $(\sim 5 \%)$. To understand the isomer impact on PFAS interaction with BSA, an additional resonance peak at $-71.868 \mathrm{ppm}$, labeled (I), was monitored throughout the CSP analysis. This resonance peak corresponds to the most abundant isopropyl branched isomer found in both PFHxS and PFOS based on spectra peak integration. Figure 9 shows the structure of the isopropyl isomer determined by previous studies. ${ }^{33,38,39}$ The carbon chain length of the PFHxS and PFOS isomer is reduced by one and the presence of a branched fluoromethyl group on the carbon adjacent to the sulfonic head group has the potential to modify the interaction of the PFAS isomer molecule with BSA in comparison with linear structures of PFHxS and PFOS.
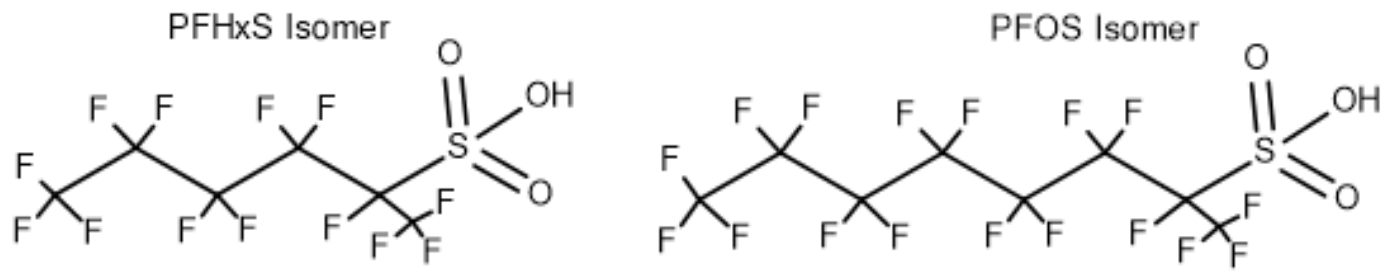

Figure 9: Structure of the isopropyl isomers of PFHxS and PFOS determined by previous studies. ${ }^{23-25}$

PFHxS exhibited a significant ${ }^{19} \mathrm{~F}$ chemical shift and broadening in both $\alpha, \Omega$ and I resonances upon binding to BSA. The $\alpha, \Omega$ and I peaks were detectable down to 2.5:1 PFHxS:BSA, indicating that below this ratio all of the PFHxS molecules are bound to BSA. The dependencies of the inverse chemical shift, $1 / \Delta \delta$, of $\alpha, \Omega$ and I resonance peaks on concentration of PFHxS in solution with $10 \mu \mathrm{M}$ BSA measured at 
three different temperatures, 298 K, 304 K, and 310 K, are shown in Figure 10. The dependencies of the chemical shift, $\Delta \delta$, of $\alpha, \Omega$ and I NMR peaks on concentration of PFHxS in solution with $10 \mu \mathrm{M}$ BSA are shown in Figure 11. Figure 10 show that in case of PFHxS, the values of the chemical shift for the isomer peak, I, are not significantly different from the $\alpha$ and $\Omega$ peaks for the linear PFHxS molecules. Figure 11 and Figure S2 show the return of the chemical shift for the $\alpha, \Omega$ and I peaks with increasing PFHxS:BSA. The chemical shifts of the $\alpha, \Omega$ and I peaks approach their original positions at 25:1 PFHxS:BSA. PFHxS was the only compound to have both peaks return to their original positions at ratios this low. This suggests that fewer PFHxS molecules bind through electrostatic forces between its head group and the charged surface area of BSA. A possible explanation stems from PFHxS having a higher water solubility than the other three PFASs studied, reflecting weaker hydrophobic interactions. ${ }^{1}$ 


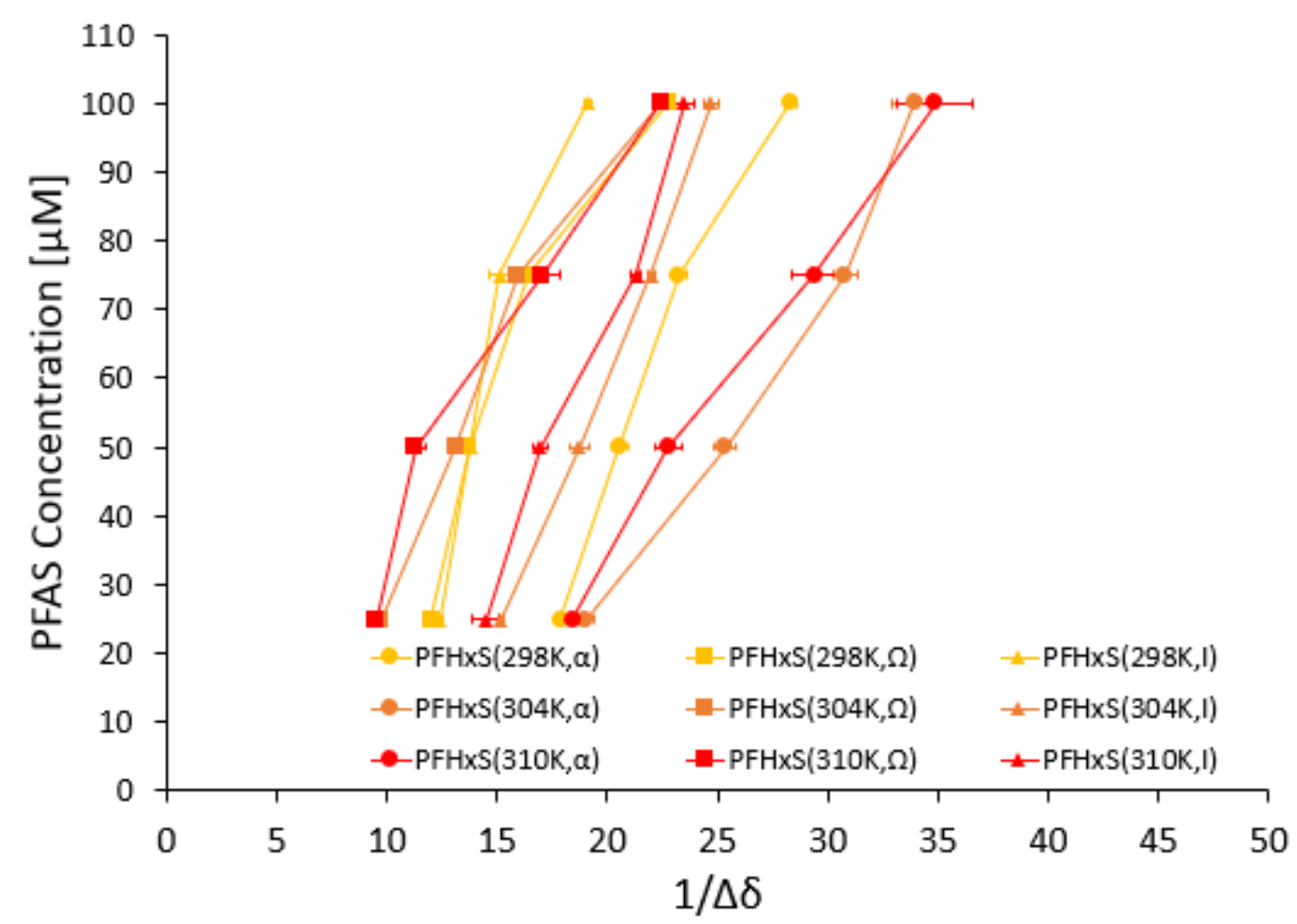

Figure 10: Dependence of the inverse chemical shift (1/ $\Delta \delta)$ of $\alpha$ (circles), $\Omega$ (squares) and I (triangles) peaks on concentration of PFHxS measured at $298 \mathrm{~K}, 304 \mathrm{~K}$, and 310 K. $\mathrm{R}^{2} \geq 0.95$ for PFHxS at all three temperatures.

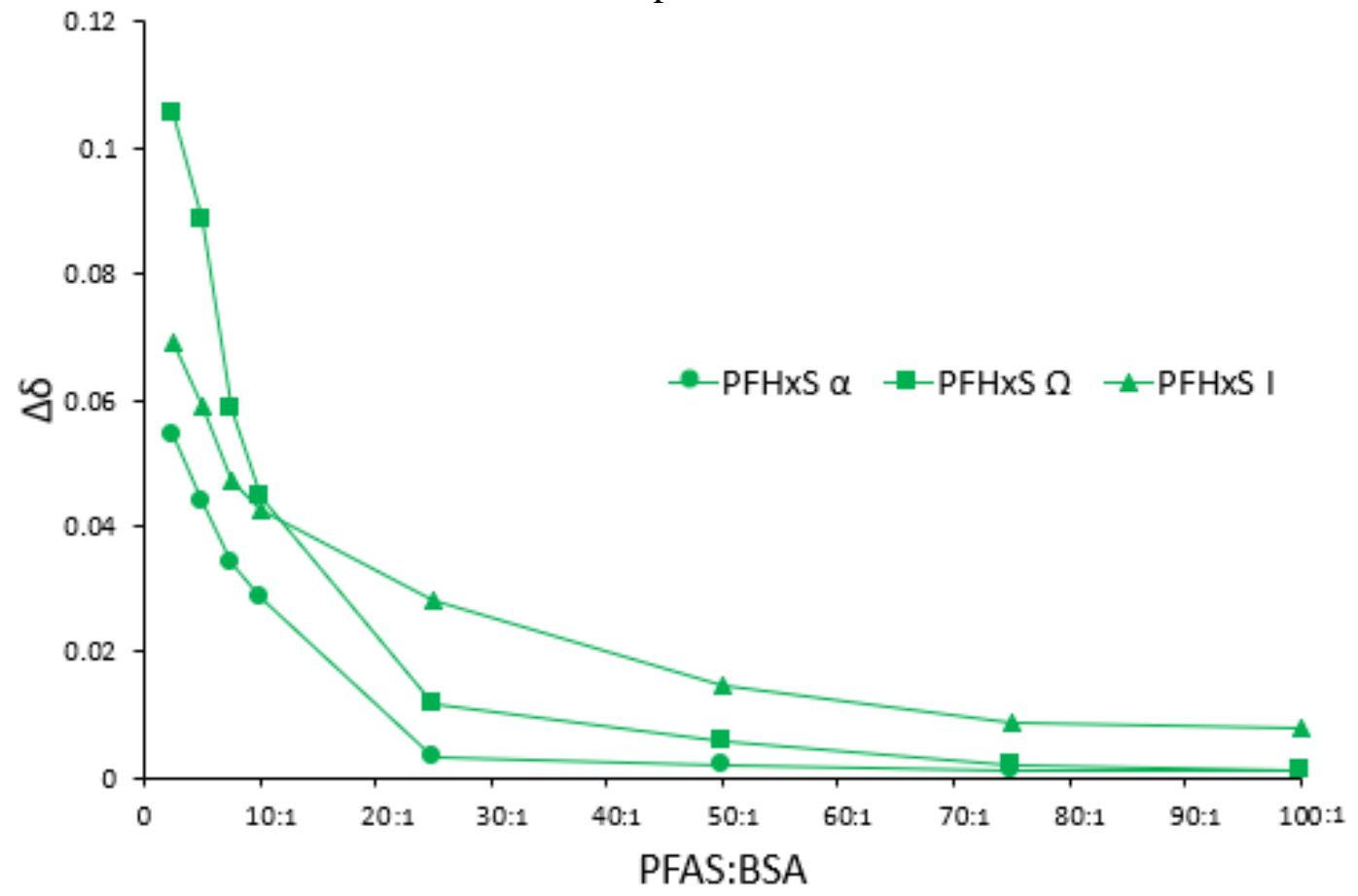

Figure 11: Dependencies of the chemical shift, $\Delta \delta$, of $\alpha$ (circles), $\Omega$ (squares) and I (triangles) peaks on PFHxS concentration with $10 \mu \mathrm{M}$ BSA measured at $310 \mathrm{~K}$. 
PFOS exhibited a significant ${ }^{19} \mathrm{~F}$ chemical shift and broadening in $\alpha, \Omega$ and I resonances upon binding to BSA. The $\alpha, \Omega$ and I peaks were detectable down to $25: 1$ PFOS:BSA. Indicating below this ratio all PFOS molecules are bound to BSA. The disappearance of the PFOS peaks was an order of magnitude higher than the other three PFASs studied. Possibly, due to the signal intensity being two orders of magnitudes less compared to the other three PFASs, because of significant isomer content. The dependencies of the inverse chemical shift $(1 / \Delta \delta)$ of $\alpha, \Omega$ and I resonance peaks on concentration of PFOS in solution with $10 \mu \mathrm{M}$ BSA measured at three different temperatures, 298K, 304K, and 310K, are seen in Figure 12. The dependencies of the chemical shift, $\Delta \delta$, of $\alpha, \Omega$ and I peaks on concentration of PFOS in solution with $10 \mu \mathrm{M}$ BSA are shown in Figure 13. Figure 12 shows that at the same concentration of PFOS the isomer peak, I, has the larger chemical shift, $\Delta \delta$, in comparison with $\alpha$ and $\Omega$ peaks, corresponding to the linear PFOS molecules. PFOS peaks contained a significant chemical shift up to 100:1 PFOS:BSA, as seen in Figure 13 and Figure S2. PFOS was the only compound to have neither $\alpha, \Omega$ and I peaks return to their original shifts as seen in Figure 13; this could be due to the large percent of isomers present causing there to be less free linear PFOS in solution with BSA. ${ }^{(28)}$ Further work on isomer effects on PFAS binding is necessary to better understand their impact on PFAS-BSA binding. 


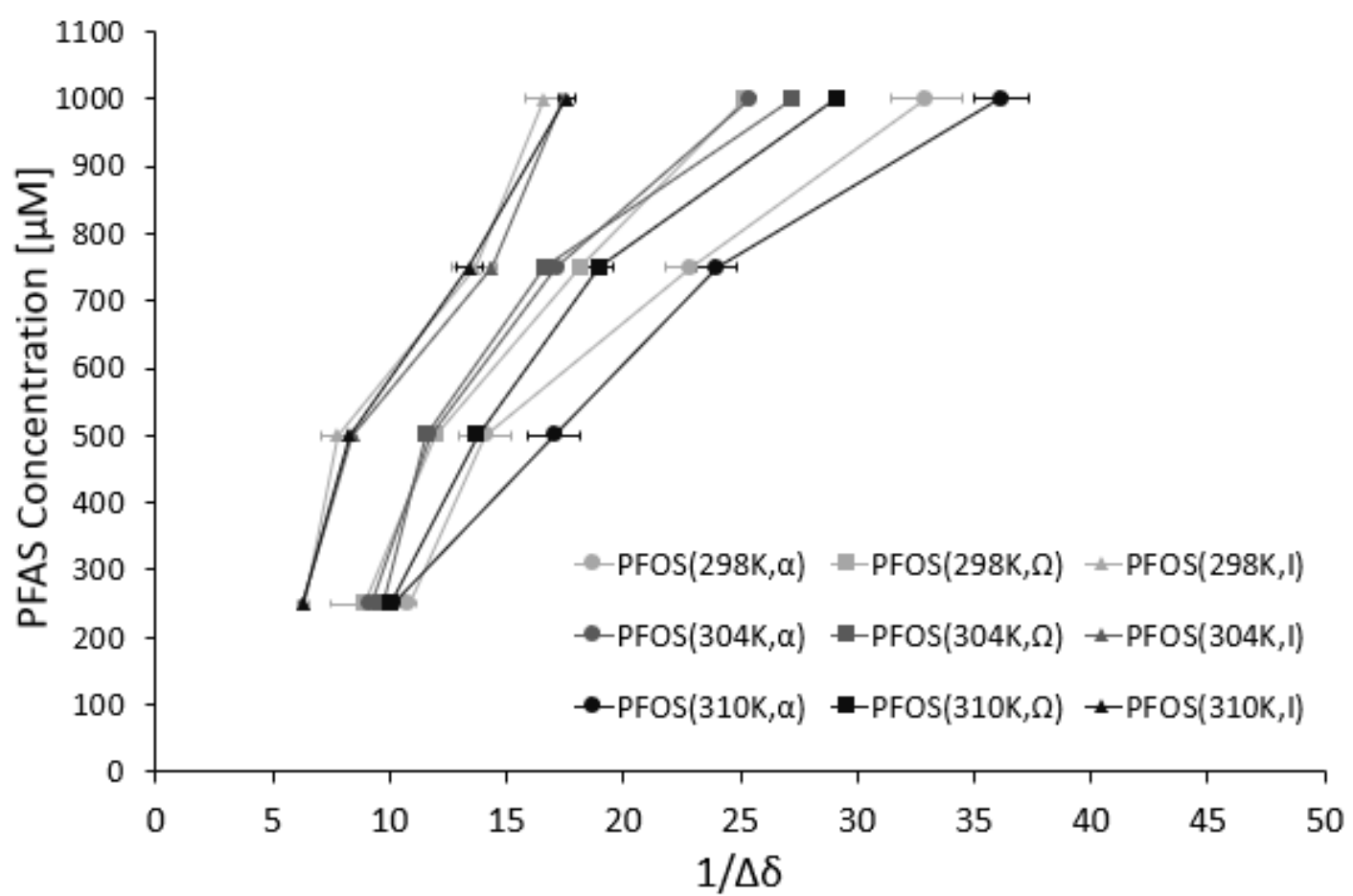

Figure 12: Dependence of the inverse chemical shift (1/ $\Delta \delta)$ of $\alpha$ (circles), $\Omega$ (squares) and I (triangles) peaks on concentration of PFOS measured at $298 \mathrm{~K}, 304 \mathrm{~K}$, and 310 K. $\mathrm{R}^{2} \geq 0.95$ for PFOS at all three temperatures.

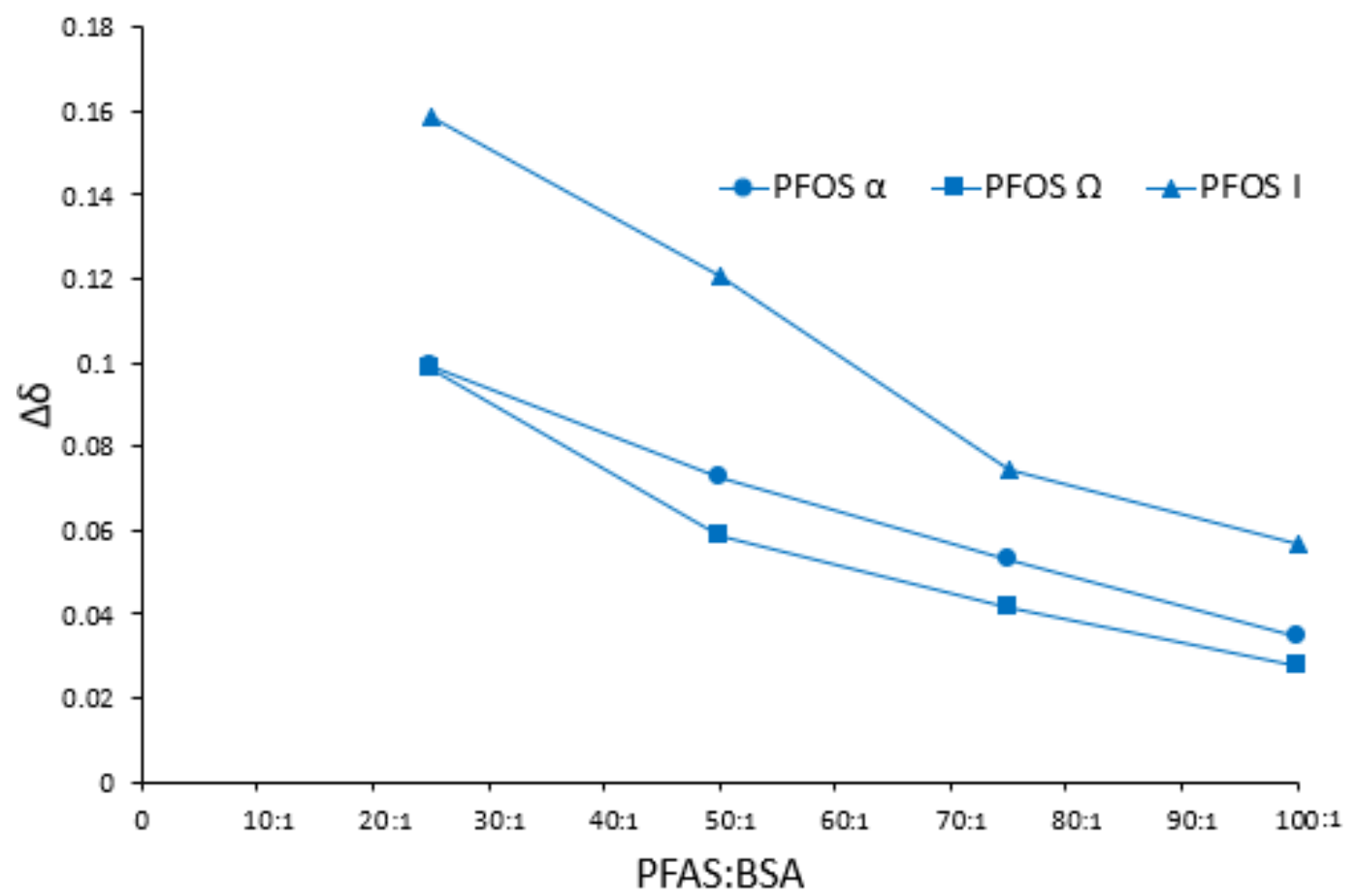

Figure 13: Dependencies of the chemical shift, $\Delta \delta$, of $\alpha$ (circles), $\Omega$ (squares) and I (triangles) peaks on PFOS concentration with $10 \mu \mathrm{M}$ BSA measured at $310 \mathrm{~K}$. 
Figure 14 shows the graphically determined $\mathrm{K}_{\mathrm{d}}$ for the interaction of the head $(\alpha)$ and tail $(\Omega)$ of linear molecules of PFHxS and PFOS as well as the isopropyl isomers (I) of PFHxS and PFOS with BSA, measured at $298 \mathrm{~K}, 304 \mathrm{~K}$ and $310 \mathrm{~K}$. All $\mathrm{K}_{\mathrm{d}}$ values are in $10^{-5} \mathrm{M}$ range, except for $\Omega$-type binding of PFOS at temperatures greater than $300 \mathrm{~K}$, the $\mathrm{K}_{d}$ was reaching values as low as $10^{-6} \mathrm{M}$. Having a $\mathrm{K}_{d}$ near $10^{-6} \mathrm{M}$, makes PFOS a possible ligand competitor with fatty acids binding with BSA. It is concluded that the $\Omega$-type binding provided the lowest $\mathrm{K}_{\mathrm{d}}$ and the highest affinity of PFHxS and PFOS binding with BSA, similar to previous studies. ${ }^{17,20,25}$ Comparing the binding of PFHxS-BSA and PFOS-BSA with each other revealed that PFOS has the higher affinity with BSA than PFHxS through $\Omega$-type interaction while their affinities with BSA are comparable in case of $\alpha$-type interaction.

A decrease in $K_{d}$ with increasing temperature indicates that hydrophobic forces are the strongest in binding of PFHxS and PFOS as well as their isopropyl isomers with BSA. Higher values of $\mathrm{K}_{\mathrm{d}}$ for the isomers of PFHxS and PFOS indicate that the steric hindrance of the branched isomer head decreases the affinity of the isomers with the surface of BSA in comparison with the linear structure of PFHxS and PFOS molecules (Figure 14). The branched isomer $\mathrm{K}_{\mathrm{d}}$ values decrease less with increase in temperature compared to the linear PFHxS and PFOS molecules. Possibly due to the hydrophobic tail shortening by one carbon compared to the linear structures, causing there to be less contribution of the hydrophobic forces to binding with BSA. 


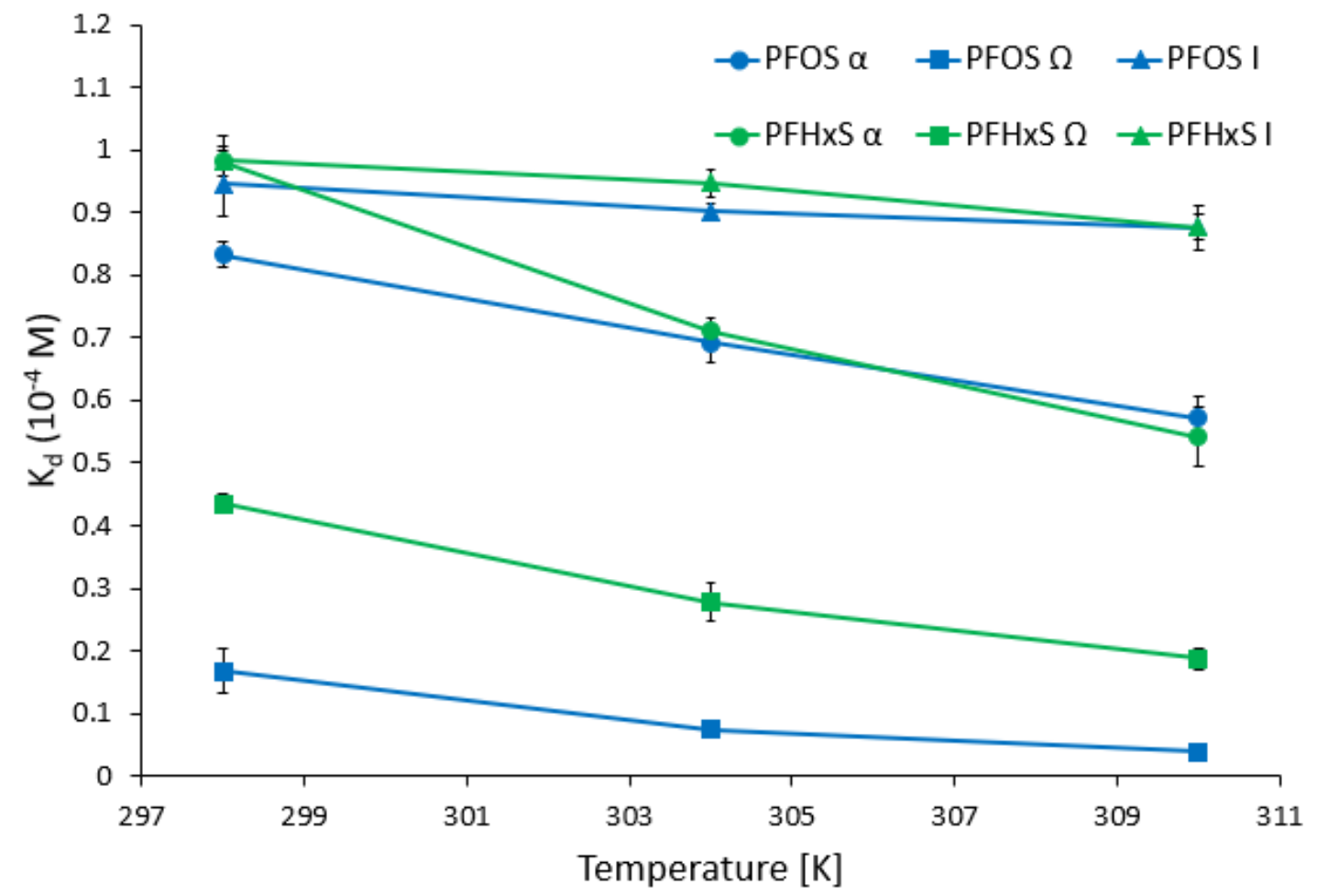

Figure 14: Dissociation constants $\left(\mathrm{K}_{\mathrm{d}}\right)$ for $\alpha$ and $\Omega$ mechanisms of binding of PFHxS (green) and PFOS (blue) and their isopropyl isomers (I) binding with BSA measured at $298 \mathrm{~K}, 304 \mathrm{~K}$, and $310 \mathrm{~K}$. Error bars are shown for each compound based on $\mathrm{n}=3$.

\subsection{Thermodynamic Parameters of BSA-Binding (PFOA, PFNA, PFHxS and PFOS)}

$\mathrm{K}_{\mathrm{d}}$ is a thermodynamic parameter characterizing the affinity of a ligand bound to a protein molecule. The values of $\Delta \mathrm{H}$ and $\Delta \mathrm{S}$ for the $\alpha$ and $\Omega$ binding mechanisms are determined from the slope $(-\Delta \mathrm{H} / \mathrm{R})$ and $\mathrm{y}$-intercept $(\Delta \mathrm{S} / \mathrm{R})$ of the plot of the linear van’t Hoff equation (6) (Figures S3 and S4). $\Delta \mathrm{G}$ is calculated using equation (5) for the $\alpha$ and $\Omega$ binding mechanisms of the PFASs with BSA. It was found that both $\Delta H$ and $\Delta \mathrm{S}$ were negative and $\Delta \mathrm{G}$ was positive for all four PFASs studied, shown in Figure 15 for the carboxylates (PFOA and PFNA) and Figure 16 for the sulfonates (PFHxS and PFOS). Negative values of $\Delta \mathrm{H}$ and $\Delta \mathrm{S}$ indicate that intermolecular hydrogen bonds and van der Waals' forces are dominating the interaction between PFASs and BSA, similar to findings from previous studies. ${ }^{34-37}$ The combination of a 
negative $\Delta \mathrm{H}$ and $\Delta \mathrm{S}$ and a positive $\Delta \mathrm{G}$ is characteristic for the interaction of water and a hydrophobic molecule with a long carbon chain. This is common in biological reactions involving hydrophobic binding with each other that are controlled by the values of $\Delta \mathrm{H}$ and $\Delta \mathrm{S}$ for the reaction. ${ }^{44}$

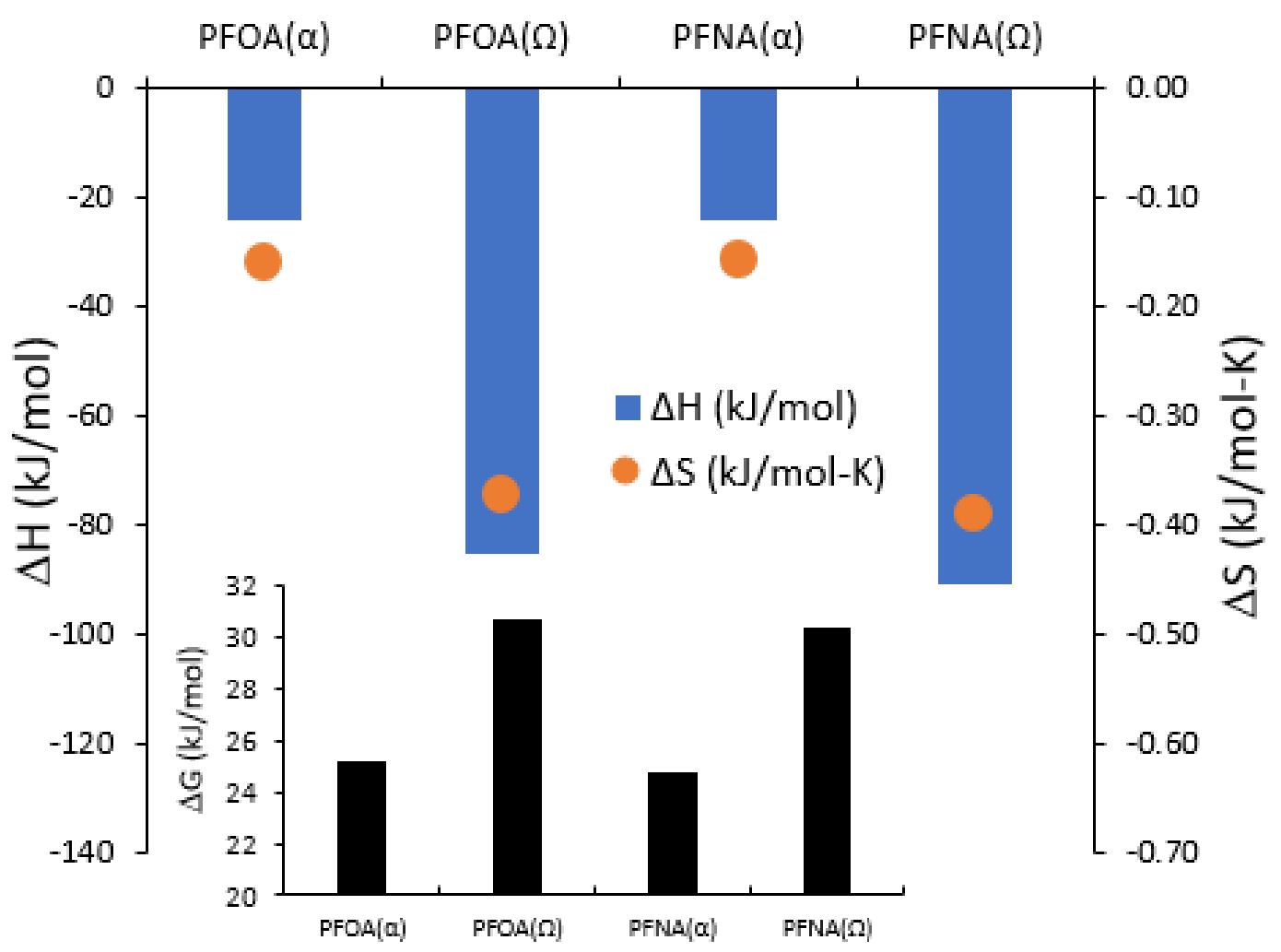

Figure 15: Enthalpy $(\Delta \mathrm{H})$, entropy $(\Delta \mathrm{S})$ and free energy $(\Delta \mathrm{G})$ for the $\alpha$ and $\Omega$ mechanisms of binding for PFOA and PFNA with BSA from 298-310 K. 


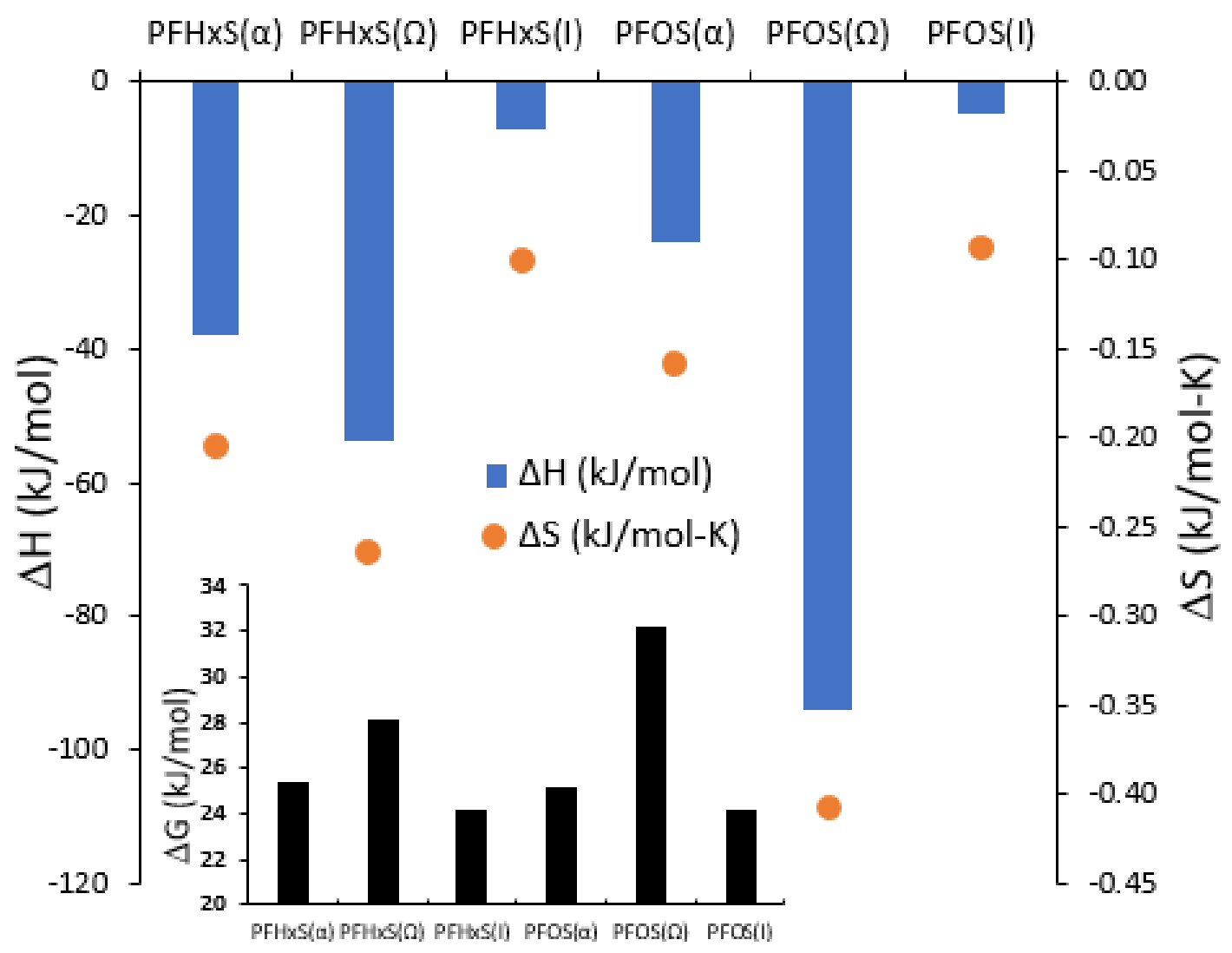

Figure 16: Enthalpy $(\Delta \mathrm{H})$, entropy $(\Delta \mathrm{S})$ and free energy $(\Delta \mathrm{G})$ for the $\alpha, \Omega$ and I mechanisms of binding for PFHxS and PFOS with BSA from 298-310 K.

Simultaneous evaluation of both $\Delta \mathrm{H}$ and $\Delta \mathrm{S}$ from only a van't Hoff plot using equation (6) can give erroneous results due to the enthalpy-entropy compensation effect. Enthalpy-entropy compensation (EEC) is a well-known phenomenon manifested in many chemical and biochemical systems. Linear plots of $\Delta \mathrm{H}$ versus $\Delta \mathrm{S}$ are often treated as authentic representations of a thermodynamic relationship, or an EEC effect. ${ }^{(45)}$ Errors can arise by the long extrapolation of the linear plot by an unknown law to get the y-intercept for $\Delta \mathrm{S}$, especially in biological systems with weak intermolecular interactions.

To check the PFAS-BSA binding thermodynamic parameters for the EEC effect as a result of errors in extrapolation of $\Delta \mathrm{S}$ from the van't Hoff plot of equation 
(6) an additional method to calculate $\Delta \mathrm{S}$ is used. $\Delta \mathrm{H}$ can still be obtained from the van't Hoff plot of equation (6) as the temperature range is narrow. The $\Delta \mathrm{G}$ is calculated from equation (4) with subsequent calculation of $\Delta \mathrm{S}$ using equation (5). Additional measurements of $\Delta \mathrm{G}$ and $\Delta \mathrm{H}$ through independent isothermal titration calorimetry (ITC) may provide more accurate evaluation of the thermodynamic parameters of the molecular interactions between PFAS and BSA.

Comparison of the $\Delta \mathrm{S}$ calculated using equation (4) and equation (5) with $\Delta \mathrm{S}$ received from van't Hoff plot of equation (6) seen in Table 1 show that they are almost identical for the $\alpha$ and $\Omega$ mechanisms of binding for PFOA, PFNA, PFHxS and PFOS with BSA. A good match of $\Delta \mathrm{S}$ calculated by two different methods in Table 1 indicates that the thermodynamic parameters of the PFAS binding with BSA defined in this work were not compromised by the EEC effect.

The thermodynamic parameters found in this study characterizing the binding of PFOA, PFNA, PFHxS and PFOS with BSA are in agreement with published data received by different techniques. ${ }^{13,26,27,28}$ 


\begin{tabular}{|c|c|c|c|c|c|c|}
\hline & & \multicolumn{2}{|c|}{ Method 1} & \multicolumn{2}{|c|}{ Method 2} \\
\hline & & $\begin{array}{c}\Delta \mathrm{H} \\
(\mathrm{kJ} / \mathrm{mol})\end{array}$ & $\begin{array}{c}\Delta \mathrm{S} \\
(\mathrm{kJ} / \mathrm{mol}-\mathrm{K})\end{array}$ & $\begin{array}{c}\Delta \mathrm{G} \\
(\mathrm{kJ} / \mathrm{mol})\end{array}$ & $\begin{array}{c}\Delta \mathrm{S} \\
(\mathrm{kJ} / \mathrm{mol}-\mathrm{K})\end{array}$ & $\begin{array}{c}\Delta \mathrm{G} \\
(\mathrm{kJ} / \mathrm{mol})\end{array}$ \\
\hline \multirow{2}{*}{ PFOA } & $(\alpha)$ & -24.260 & -0.159 & 25.173 & -0.160 & 25.213 \\
\hline & $(\Omega)$ & -84.989 & -0.373 & 30.730 & -0.373 & 30.787 \\
\hline \multirow{2}{*}{ PFNA } & $(\alpha)$ & -24.205 & -0.158 & 24.813 & -0.158 & 24.805 \\
\hline & $(\Omega)$ & -90.778 & -0.391 & 30.370 & -0.391 & 30.357 \\
\hline \multirow{3}{*}{ PFHxS } & $(\alpha)$ & -37.985 & -0.204 & 25.335 & -0.204 & 25.317 \\
\hline & $(\Omega)$ & -53.922 & -0.264 & 28.070 & -0.264 & 28.053 \\
\hline & (I) & -7.264 & -0.101 & 24.059 & -0.101 & 24.078 \\
\hline \multirow{3}{*}{ PFOS } & $(\alpha)$ & -24.094 & -0.159 & 25.174 & -0.159 & 25.180 \\
\hline & $(\Omega)$ & -94.127 & -0.407 & 32.184 & -0.407 & 32.124 \\
\hline & (I) & -4.913 & -0.094 & 24.087 & -0.094 & 24.080 \\
\hline
\end{tabular}

Table 1. Thermodynamic parameters, enthalpy $(\Delta H)$, entropy $(\Delta S)$ and Gibb's free energy $(\Delta \mathrm{G})$ characterizing the interaction of PFOA, PFNA, PFHxS and PFOS with BSA in PBS buffer at pH 7.4 defined from linear van't Hoff plot only (Method 1) and from the combination of van't Hoff plot and $K_{d}=e^{-\frac{\Delta G}{R T}}($ Method 2). 


\section{CHAPTER 4}

\section{CONCLUSION}

This study performed a comparative investigation of the thermodynamic binding interactions between four PFAS molecules (PFOA, PFNA, PFHxS and PFOS) with BSA using ${ }^{19} \mathrm{~F}$ NMR spectroscopy, with synchronous observation of the ${ }^{19} \mathrm{~F}$ signals from both ends of the PFAS molecules. Chemical shifts of ${ }^{19} \mathrm{~F}$ NMR peaks in solutions of PFAS with BSA were used to evaluate the equilibrium dissociation constants, $\mathrm{K}_{\mathrm{d}}$, for both known mechanisms of PFAS-BSA binding: by hydrophobic interactions of the PFAS molecule tail with hydrophobic pockets in the BSA core $(\Omega$ mechanism) and by the hydrogen bonds and electrostatic interactions of the PFAS molecule head group with charged regions on the BSA surface ( $\alpha$ mechanism).

The highest affinity of the PFAS-BSA complexes is by the $\Omega$ mechanism of binding, with $\mathrm{K}_{\mathrm{d}}$ reaching as low as $3.9 \times 10^{-6}, 6.5 \times 10^{-6}, 7.7 \times 10^{-6}$ and $1.9 \times 10^{-5} \mathrm{M}$ for PFOS, PFOA, PFNA and PFHxS, in comparison with $5.7 \times 10^{-5}, 5.6 \times 10^{-5}, 6.6 \times 10^{-5}$ and $5.4 \times 10^{-5} \mathrm{M}$ for the $\alpha$ mechanism of binding. The $\mathrm{K}_{\mathrm{d}}$ follow a U-shape with increase in chain length. Demonstrating that PFASs with tails greater than 8 carbons begin to decrease in binding affinity with BSA. This U-shape trend has been reported for placental transfer efficiencies (PTE) of PFASs, indicating that $\mathrm{K}_{\mathrm{d}}$ can be accurately correlated with PTE. ${ }^{(23)}$ These values also suggest that PFASs have a stronger binding affinity than previously thought by other studies and they may have the ability to displace natural fatty acids from the hydrophobic pockets of BSA. Further 
investigation into this two-step binding sequence and its effect on PTE, as well the ability of PFASs to displace natural fatty acids in physiological conditions should be performed.

The $K_{d}$ for the $\alpha$ mechanism of binding within an order of magnitude of the $\Omega$ mechanism of binding suggests that both carboxylic and sulfonate head groups have a strong binding affinity. This is not seen in their hydrocarbon analogs that primarily rely on their hydrophobic tail to bind with BSA. The ability of the PFAS head group to reversibly bind with BSA should be looked at more closely, to better comprehend their physiological effects and how they are transported throughout physiological systems. Additionally, determining a single $\mathrm{K}_{\mathrm{d}}$ for a PFAS based on both $\alpha$ and $\Omega$ mechanism of binding, such as equation (9) should be investigated to better predict their binding affinity and PTE, such as the relationship seen below.

$$
\mathrm{K}_{\mathrm{d}}=\frac{[\mathrm{PFAS}][\mathrm{BSA}]}{[\mathrm{PFAS}-\mathrm{BSA}]_{\alpha}}+\frac{[\mathrm{PFAS}][\mathrm{BSA}]}{[\mathrm{PFAS}-\mathrm{BSA}]_{\Omega}}
$$

Evaluation of the thermodynamic parameters enthalpy $(\Delta \mathrm{H})$, entropy $(\Delta \mathrm{S})$ and Gibb's free energy $(\Delta G)$ showed that binding of PFOA, PFNA, PFHxS and PFOS with BSA by both $\alpha$ and $\Omega$ mechanisms is accompanied by a negative $\Delta \mathrm{H}$ and $\Delta \mathrm{S}$ and a positive $\Delta \mathrm{G}$, which is characteristic for the binding of two large hydrophobic molecules by weak hydrogen bonds and van der Waals' forces. Additional experiments using isothermal titration calorimetry (ITC) should be performed to accurately determine and compare thermodynamic parameters of PFAS-BSA binding.

The $\mathrm{K}_{\mathrm{d}}$ for the binding of the branched isopropyl isomers of PFHxS and PFOS with BSA were measured for the first time. The isopropyl isomers of PFHxS and PFOS had a $\mathrm{K}_{\mathrm{d}}$ of $8.8 \times 10^{-5}$ and $7.6 \times 10^{-5} \mathrm{M}$, showing a lower affinity with the surface 
of BSA in comparison with the linear structure of the molecules, possibly due to steric hindrance of the branched isomer head. Further investigation into isomer PFAS-BSA binding is needed to better understand their impacts in physiological systems.

Additional studies on the ability of BSA to stabilize PFAS micelle formation and aggregation should be performed. Limited work has been published in this area and many PFASs still lack reported physiochemical property data. ${ }^{19} \mathrm{~F}$ NMR is a powerful tool to get high throughput results for a large quantity of PFASs.

Over the last decade, ${ }^{19} \mathrm{~F}$ NMR equipment and software has advanced greatly allowing experiments to be performed at lower concentrations, with larger more complicated proteins. ${ }^{19} \mathrm{~F}$ NMR should become a standard procedure when analyzing the ability of PFASs to bind and how their properties are affected in various systems. Future work will be dedicated to multiple peak analysis programs and peak decoupling programs, such as PeakFit and Originlab, which are useful in analyzing a large number of ${ }^{19} \mathrm{~F}$ NMR peaks at a rapid rate. A good rule when attempting to perform any PFASprotein binding experiment is: (1) if working with complex protein(s) use a simple linear PFAS and (2) if working with a complex PFAS use a small single binding site protein, as to not complicate the system to where it becomes difficult to understand the results of the ${ }^{19} \mathrm{~F}$ NMR spectra. 


\section{APPENDIX}

\section{Supplementary Data}

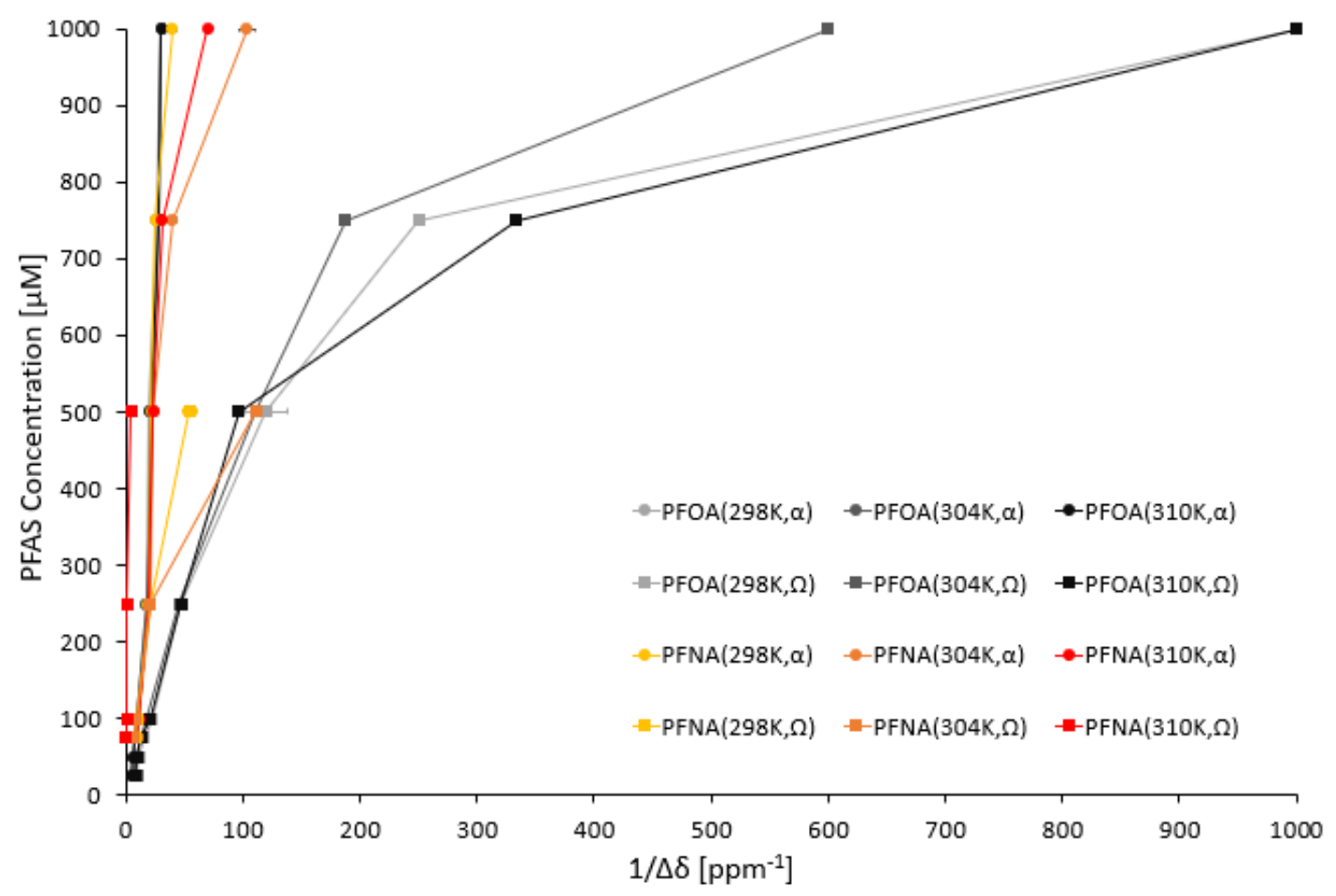

Figure S1: Chemical Shift Perturbation Data for PFOA and PFNA across entire concentration range.

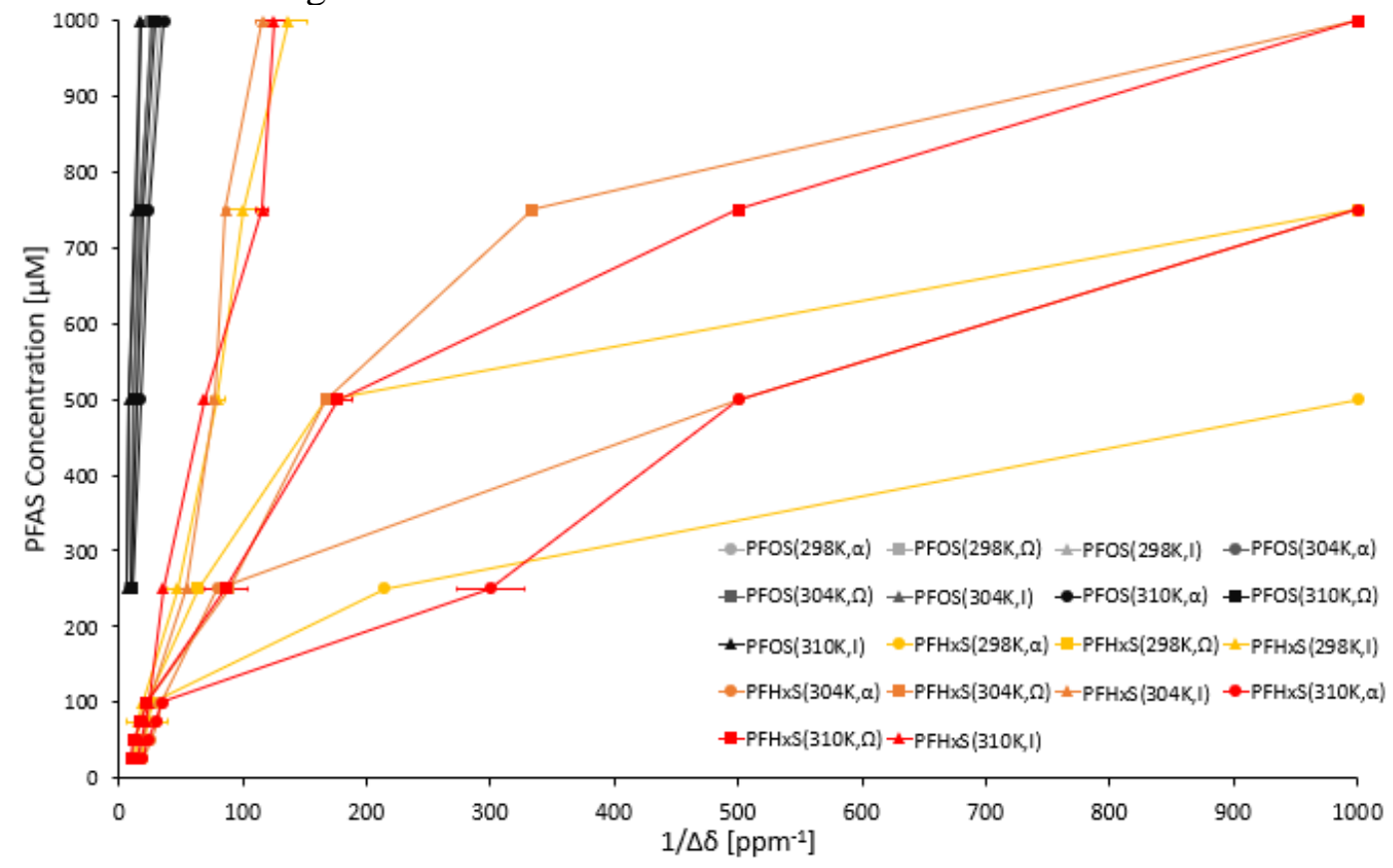

Figure S2: Chemical Shift Perturbation Data for PFHxS and PFOS across entire concentration range. 


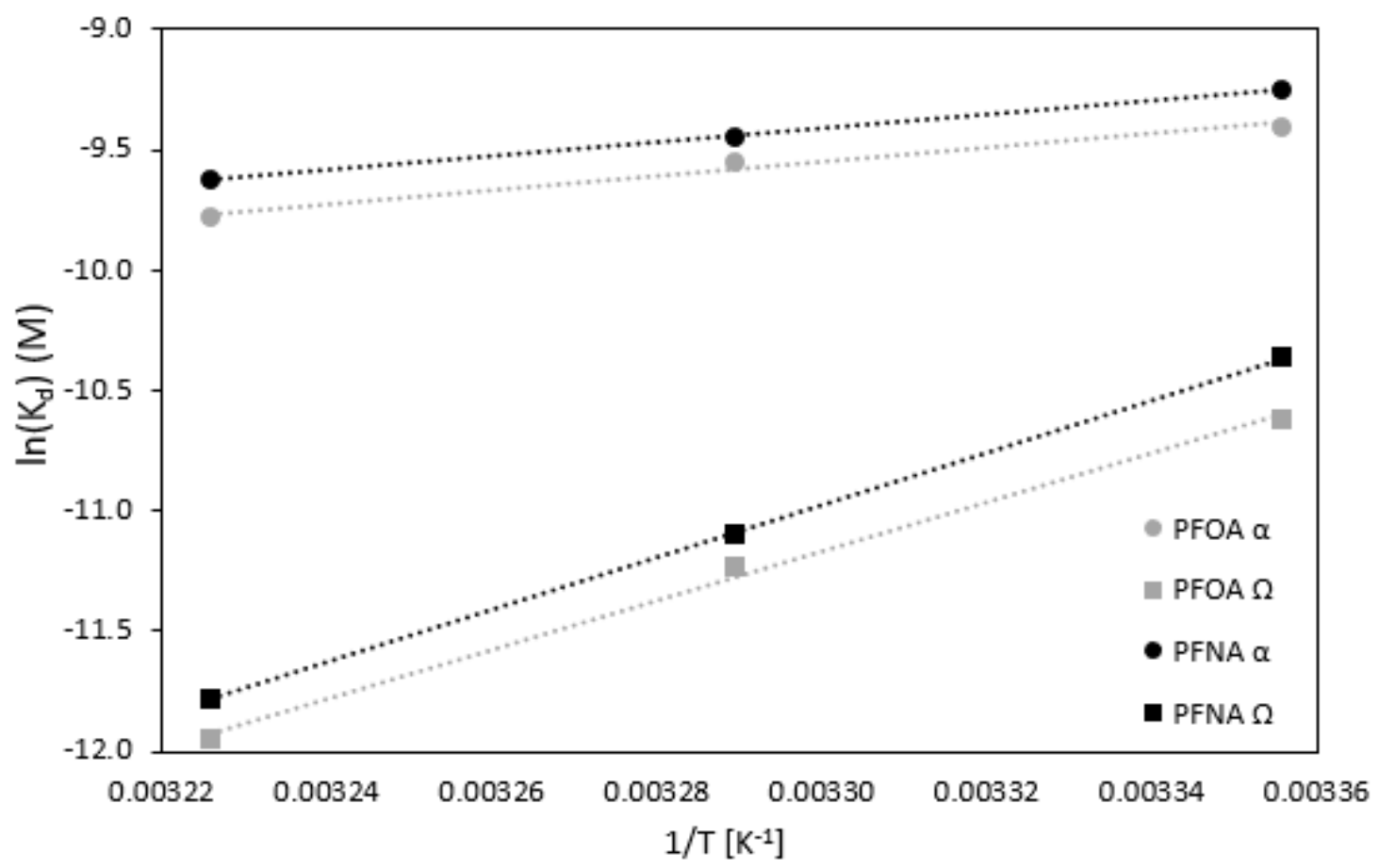

Figure S3: Thermodynamic analysis using van’t Hoff Equation for Carboxylic Acids, all $\mathrm{R}^{2} \geq 0.95$.

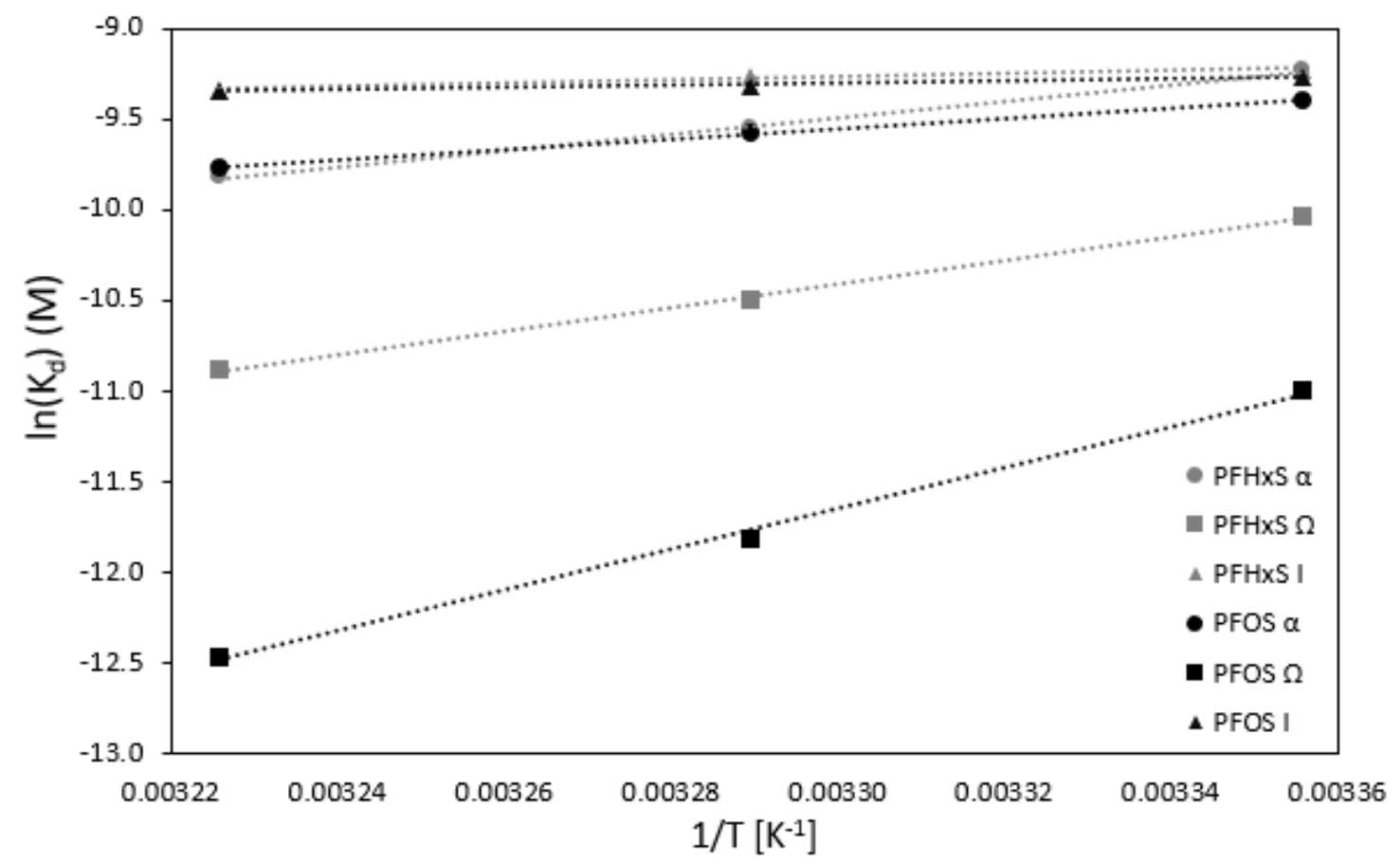

Figure S4: Thermodynamic analysis using van't Hoff Equation for Sulfonic Acids, all $\mathrm{R}^{2} \geq 0.95$. 


\section{BIBLIOGRAPHY}

1. Health and Ecological Criteria Division. "Health Effects Support Document for Perfluorooctanoic Acid (PFOA).” U.S. Environmental Protection Agency, 2016, pp. 1-322., doi:10.1289/ehp.0901827.

2. Health and Ecological Criteria Division. "Drinking Water Health Advisory for Perfluorooctanoic Acid (PFOA)." U.S. Environmental Protection Agency, 2016, pp. 1-103., doi:10.3390/w11102003.

3. Zainuddin, Khairunnisa, et al. "Perfluorooctanoic Acid (PFOA) and Perfluorooctane Sulfonate (PFOS) in Surface Water from the Langat River, Peninsular Malaysia." Environmental Forensics, vol. 13, no. 1, 2012, pp. 82-92., doi:10.1080/15275922.2011.643335.

4. Mogensen, Ulla B., et al. "Breastfeeding as an Exposure Pathway for Perfluorinated Alkylates.” Environmental Science \& Technology, vol. 49, no. 17, 2015, pp. 10466-10473., doi:10.1021/acs.est.5b02237.

5. Zhang, Tao, et al. "PFOS and PFOA in Paired Urine and Blood from General Adults and Pregnant Women: Assessment of Urinary Elimination.” Environmental Science and Pollution Research, vol. 22, no. 7, 2014, pp. 55725579., doi:10.1007/s11356-014-3725-7.

6. Kärrman, Anna, et al. "Exposure of Perfluorinated Chemicals through Lactation: Levels of Matched Human Milk and Serum and a Temporal Trend, 19962004, in Sweden.” Environmental Health Perspectives, vol. 115, no. 2, 2007, pp. 226-230., doi:10.1289/ehp.9491. 
7. Simard, Jeffrey R., et al. "Location of High and Low Affinity Fatty Acid Binding Sites on Human Serum Albumin Revealed by NMR Drug-Competition Analysis.” Journal of Molecular Biology, vol. 361, no. 2, 2006, pp. 336-351., doi:10.1016/j.jmb.2006.06.028.

8. Olsen, Geary W., et al. "Half-Life of Serum Elimination of Perfluorooctanesulfonate, Perfluorohexanesulfonate, and Perfluorooctanoate in Retired Fluorochemical Production Workers.” Environmental Health Perspectives, vol. 115, no. 9, 2007, pp. 1298-1305., doi:10.1289/ehp.10009.

9. Macneil, Jessica, et al. “A Cross-Sectional Analysis of Type II Diabetes in a Community with Exposure to Perfluorooctanoic Acid (PFOA).”

Environmental Research, vol. 109, no. 8, 2009, pp. 997-1003., doi:10.1016/j.envres.2009.08.002.

10. Grandjean, Philippe, et al. "Serum Vaccine Antibody Concentrations in Children Exposed to Perfluorinated Compounds." Journal of the American Medical Association, vol. 307, no. 4, 2012, doi:10.1001/jama.2011.2034.

11. Darrow, Lyndsey A., et al. "Serum Perfluorooctanoic Acid and Perfluorooctane Sulfonate Concentrations in Relation to Birth Outcomes in the Mid-Ohio Valley, 2005-2010.” Environmental Health Perspectives, vol. 121, no. 10, 2013, pp. 1207-1213., doi:10.1289/ehp.1206372.

12. Fletcher, Tony. "The Science Panel Website.” C8 Science Panel Website, 2013, www.c8sciencepanel.org/index.html.

13. Raleigh, Katherine K, et al. "Mortality and Cancer Incidence in Ammonium Perfluorooctanoate Production Workers.” Occupational and Environmental 
Medicine, vol. 71, no. 7, 2014, pp. 500-506., doi:10.1136/oemed-2014102109.

14. Sun, Mei, et al. "Legacy and Emerging Perfluoroalkyl Substances Are Important Drinking Water Contaminants in the Cape Fear River Watershed of North Carolina.” Environmental Science \& Technology Letters, vol. 3, no. 12, 2016, pp. 415-419., doi:10.1021/acs.estlett.6b00398.

15. Reo, Nicholas. "Hepatic Metabolism of Perfluorinated Carboxylic Acids: A Nuclear Magnetic Resonance Investigation.” Air Force Office of Scientific Research, vol.1, no. 1, 1990, pp. 1-9., doi:10.1016/b978-012523475-7/501741.

16. Jones, Paul D., et al. "Binding of Perfluorinated Fatty Acids To Serum Proteins." Environmental Toxicology and Chemistry, vol. 22, no. 11, 2003, p. 26392649., doi:10.1897/02-553.

17. Nordby, Gordon., et al. "Perfluorooctanoic Acid Interacts with Human Serum Albumin.” Journal of Molecular Biology, vol. 219, no. 1, 1956, pp. 399-404., doi:10.1126/science.124.3215.266.

18. D'eon, Jessica C., et al. "Determining the Molecular Interactions of Perfluorinated Carboxylic Acids with Human Sera and Isolated Human Serum Albumin Using Nuclear Magnetic Resonance Spectroscopy.” Environmental Toxicology and Chemistry, vol. 29, no. 8, 2010, pp. 1678-1688., doi:10.1002/etc.204.

19. Macmanus-Spencer, Laura A., et al. "Binding of Perfluorocarboxylates to Serum Albumin: A Comparison of Analytical Methods.” Analytical Chemistry, vol. 82, no. 3, 2010, pp. 974-981., doi:10.1021/ac902238u. 
20. Han, Xing, et al. "Binding of Perfluorooctanoic Acid to Rat and Human Plasma Proteins." Chemical Research in Toxicology, vol. 16, no. 6, 2003, pp. 775781., doi:10.1021/tx034005w.

21. Bischel, Heather N., et al. "Strong Associations of Short-Chain Perfluoroalkyl Acids with Serum Albumin and Investigation of Binding Mechanisms.” Environmental Toxicology and Chemistry, vol. 30, no. 11, 2011, pp. 24232430., doi:10.1002/etc.647.

22. Fielding, L. "NMR Methods for the Determination of Protein-Ligand Dissociation Constants." Progress in Nuclear Magnetic Resonance Spectroscopy, vol. 51, no. 4, 2007, pp. 219-242., doi:10.1016/j.pnmrs.2007.04.001.

23. Gao, Ke, et al. "Prenatal Exposure to Per- and Polyfluoroalkyl Substances (PFASs) and Association between the Placental Transfer Efficiencies and Dissociation Constant of Serum Proteins-PFAS Complexes." Environmental Science \& Technology, vol. 53, no. 11, 2019, pp. 6529-6538., doi:10.1021/acs.est.9b00715.

24. Ren, Xiao-Min, et al. "Binding Interactions of Perfluoroalkyl Substances with Thyroid Hormone Transport Proteins and Potential Toxicological Implications.” Toxicology, vol. 366-367, no. 1, 2016, pp. 32-42., doi:10.1016/j.tox.2016.08.011.

25. Okaru, Alex O., et al. "Application of ${ }^{19}$ F NMR Spectroscopy for Content Determination of Fluorinated Pharmaceuticals." Journal of Analytical Methods in Chemistry, vol. 2017, no. 1, 2017, pp. 1-7., doi:10.1155/2017/9206297. 
26. Sugiki, Toshihiko, et al. "Current NMR Techniques for Structure-Based Drug Discovery.” Molecules, vol. 23, no. 1, 2018, pp. 148-175., doi:10.3390/molecules23010148.

27. Moody, Cheryl A., et al. "Determination of Perfluorinated Surfactants in Surface Water Samples by Two Independent Analytical Techniques: Liquid Chromatography/Tandem Mass Spectrometry and ${ }^{19}$ F NMR.” Analytical Chemistry, vol. 73, no. 10, 2001, pp. 2200-2206., doi:10.1021/ac0100648.

28. Williamson, Mike P. "Using Chemical Shift Perturbation to Characterize Ligand Binding." Progress in Nuclear Magnetic Resonance Spectroscopy, vol. 73, no. 1, 2013, pp. 1-16., doi:10.1016/j.pnmrs.2013.02.001.

29. Hu, Xindi C., et al. "Detection of Poly- and Perfluoroalkyl Substances (PFASs) in U.S. Drinking Water Linked to Industrial Sites, Military Fire Training Areas, and Wastewater Treatment Plants." Environmental Science \& Technology Letters, vol. 3, no. 10, 2016, pp. 344-350., doi:10.1021/acs.estlett.6b00260.

30. Lath, Supriya, et al. "Sorption of PFOA onto Different Laboratory Materials: Filter Membranes and Centrifuge Tubes." Chemosphere, vol. 222, no.1, 2019, pp. 671-678., doi:10.1016/j.chemosphere.2019.01.096.

31. Shortridge, Matthew D., et al. "Estimating Protein-Ligand Binding Affinity Using High-Throughput Screening by NMR.” Journal of Combinatorial Chemistry, vol. 10, no. 6, 2008, pp. 948-958., doi:10.1021/cc800122m.

32. Swift, T. Jet al. "NMR-Relaxation Mechanisms of ${ }^{17} \mathrm{O}$ in Aqueous Solutions of Paramagnetic Cations and the Lifetime of Water Molecules in the First 
Coordination Sphere.” The Journal of Chemical Physics, vol. 41, no. 8, 1964, pp. 2553-2554., doi:10.1063/1.1726303.

33. Arsenault, Gilles, et al. "Separation and Fluorine Nuclear Magnetic Resonance Spectroscopic ( $\left({ }^{19} \mathrm{~F}\right.$ NMR) Analysis of Individual Branched Isomers Present in Technical Perfluorooctanesulfonic Acid (PFOS).” Chemosphere, vol. 73, no. 1, 2008, pp. 53-59., doi:10.1016/j.chemosphere.2007.06.096.

34. Zhang, Xian, et al. "Binding of PFOS to Serum Albumin and DNA: Insight into the Molecular Toxicity of Perfluorochemicals." BMC Molecular Biology, vol. 10, no. 1, 2009, pp. 10-16., doi:10.1186/1471-2199-10-16.

35. Gao, Sichen, et al. "Comprehensive Insights into the Interaction Mechanism between Perfluorodecanoic Acid and Human Serum Albumin.” New Journal of Chemistry, vol. 42, no. 11, 2018, pp. 9065-9073., doi:10.1039/c8nj00124c.

36. Qin, Pengfei, et al. "Impact of Carbon Chain Length on Binding of Perfluoroalkyl Acids to Bovine Serum Albumin Determined by Spectroscopic Methods.” Journal of Agricultural and Food Chemistry, vol. 58, no. 9, 2010, pp. 55615567., doi:10.1021/jf100412q.

37. Chen, Huilun, et al. "Systematic Investigation of the Toxic Mechanism of PFOA and PFOS on Bovine Serum Albumin by Spectroscopic and Molecular Modeling." Chemosphere, vol. 129, no. 1, 2015, pp. 217-224., doi:10.1016/j.chemosphere.2014.11.040.

38. Liu, P., et al. "Theoretical Studies of the Conformations and ${ }^{19}$ F NMR Spectra of Linear and a Branched Perfluorooctanesulfonamide (PFOSAmide).” 
Chemosphere, vol. 69, no. 8, 2007, pp. 1213-1220., doi:10.1016/j.chemosphere.2007.06.006.

39. Vyas, Sandhya M., et al. "Differences in the Isomer Composition of Perfluoroctanesulfonyl (PFOS) Derivatives." Journal of Environmental Science and Health, Part A, vol. 42, no. 3, 2007, pp. 249-255., doi:10.1080/10934520601134031.

40. Otting, Gottfried. "Experimental NMR Techniques for Studies of Protein-Ligand Interactions." Current Opinion in Structural Biology, vol. 3, no. 5, 1993, pp. 760-768., doi:10.1016/0959-440x(93)90061-o.

41. Zhao, Yanchuan, et al. " ${ }^{19}$ F NMR Fingerprints: Identification of Neutral Organic Compounds in a Molecular Container." Journal of the American Chemical Society, vol. 136, no. 30, 2014, pp. 10683-10690., doi:10.1021/ja504110f.

42. Salvalaglio, Matteo, et al. "Determination of Energies and Sites of Binding of PFOA and PFOS to Human Serum Albumin.” The Journal of Physical Chemistry B, vol. 114, no. 46, 2010, pp. 14860-14874., doi:10.1021/jp106584b.

43. Ellis, David A., et al. "The Use of ${ }^{19}$ F NMR to Interpret the Structural Properties of Perfluorocarboxylate Acids: A Possible Correlation with Their Environmental Disposition.” The Journal of Physical Chemistry A, vol. 108, no. 46, 2004, pp. 10099-10106., doi:10.1021/jp049372a.

44. Garrett, Reginald H., and Charles M. Grisham. Biochemistry. 5th ed., vol. 1, Cengage Learning, 2017. 
45. Khrapunov, Sergei. “The Enthalpy-Entropy Compensation Phenomenon.

Limitations for the Use of Some Basic Thermodynamic Equations." Current

Protein \& Peptide Science, vol. 19, no. 11, 2018, pp. 1088-1091., doi:10.2174/1389203719666180521092615. 